\title{
L'« ornement » en abyme ou la sémantique du détail
}

The " ornament » en abyme or the semantics of detail

\section{Nikolina Kei}

\section{(2) OpenEdition}

1 Journals

Édition électronique

URL : http://journals.openedition.org/imagesrevues/8778

DOI : 10.4000/imagesrevues.8778

ISSN : $1778-3801$

Éditeur :

Centre d'Histoire et Théorie des Arts, Groupe d'Anthropologie Historique de l'Occident Médiéval, Laboratoire d'Anthropologie Sociale, UMR 8210 Anthropologie et Histoire des Mondes Antiques

\section{Référence électronique}

Nikolina Kei, «L'« ornement » en abyme ou la sémantique du détail », Images Re-vues [En ligne], Horssérie 9 | 2020, mis en ligne le 01 décembre 2020, consulté le 16 mars 2021. URL : http://

journals.openedition.org/imagesrevues/8778; DOI : https://doi.org/10.4000/imagesrevues.8778

Ce document a été généré automatiquement le 16 mars 2021.

Images Re-vues est mise à disposition selon les termes de la Licence Creative Commons Attribution Pas d'Utilisation Commerciale 4.0 International. 


\section{L'« ornement » en abyme ou la sémantique du détail}

The « ornament " en abyme or the semantics of detail

\section{Nikolina Kei}

Je tiens à remercier Giulia Puma et Stéphanie Wyler pour m'avoir offert l'occasion de publier cette brève étude faisant partie de ma thèse de doctorat intitulée "L'esthétique des fleurs : kosmos, poikilia et kharis dans la céramique attique du vie et du ve siècle av. n. ère » (EHESS, 2010). Je suis particulièrement reconnaissante au relecteur anonyme et à Giulia Puma pour leurs remarques très justes et leurs propositions. 
1 Lorsqu'ils souhaitaient densifier le sens de leurs images, les peintres des vases attiques avaient recours à plusieurs stratégies iconographiques. Parmi ces stratégies, souvent perçues seulement grâce à un regard attentif et aiguisé, figure la mise en abyme $^{1}$ de ce qu'on appelle traditionnellement

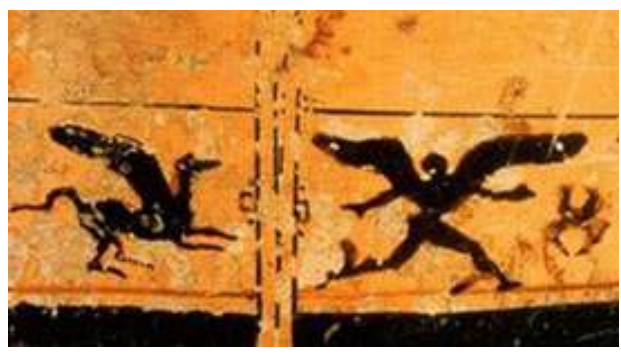
« ornements »: il s'agit de représenter de manière micrographique des motifs végétaux, animaliers, stellaires et même des figures humaines, sur des objets de luxe (vêtements, armes, meubles, vases et éléments architecturaux) meublant des scènes à caractère varié. La présence de ces motifs, censés être tissés, brodés, gravés, incisés, incrustés, peints ou sculptés, loin d'être accessoire et superflue, est d'ordre dynamique : ils opèrent à la fois comme ornements et signes polyvalents. En tant qu'ornements, ils revêtent leurs supports d'une allure somptueuse issue d'un travail soigné, allure qui les élève au-dessus de l'ordre du quotidien et du commun. En tant que signes, ils renvoient à l'ensemble de l'image, que ce soit sous forme d'écho, de répétition, d'intensification, de prolongement, d'explicitation ou de contraste.

2 Autrement dit, ces motifs discrets véhiculent et visualisent trois notions proprement grecques : la poikilia, à savoir la bigarrure ${ }^{2}$, le kosmos, au sens de parure ${ }^{3}$, et surtout la charis ${ }^{4}$, au sens de grâce resplendissante émanant d'une présence physique, trois notions qui, lorsqu'elles s'appliquent à un objet, lui assignent le statut d'un daidalon, d'un poikilon et sans doute celui d'un agalma. Les trois derniers termes exigent une clarification. Comme l'a montré Françoise Frontisi-Ducroux ${ }^{5}$, le daidalon évoque l'effet visuel issu du travail raffiné de l'artisan, et plus particulièrement du scintillement d'une surface composite et nuancée; par conséquent, il qualifie les produits de l'artisanat de luxe, signes de prestige social. Tout comme le daidalon, le poikilon dénote le chatoiement qui résulte d'un travail d'assemblage harmonieux des matières et des couleurs, qu'il s'agisse du tissage ou de la toreutique. Enfin, l'agalma est une belle chose (kalon, perikalles), pas nécessairement un artefact, qui génère du plaisir visuel et qui confère de l'honneur (kydos) à son possesseur ${ }^{6}$. Les objets au décor varié que nous examinerons dans cette brève étude, pourraient appartenir à la catégorie d'agalma, ne serait-ce que par le fait qu'ils sont censés accrocher le regard, charmer et réjouir les yeux ${ }^{7}$.

3 La mise en abyme des motifs végétaux, animaliers, stellaires et autres, pose deux questions d'ordre à la fois iconographique et sémantique: celle du rapport entre " image » et " ornement » et celle du détail. Notons d'abord que ces motifs, parce que d'échelle réduite et donc moins accessibles au regard que les autres éléments de l'image, ont été souvent négligés et cantonnés à la catégorie d'«ornement " ${ }^{8}$. Or le rapport antinomique entre ce qu'on appelle d'habitude "figure " / " ornement " ou " image " / " décor ", chaque couple étant considéré comme constitué de deux entités monolithiques, distinctes et opposées, est une fiction académique des XVIII ${ }^{\mathrm{e}}$ et $\mathrm{XIX}^{\mathrm{e}}$ siècles créée pour des raisons stylistiques et chronologiques. L'idée derrière cette polarité est simple : l'image seule est porteuse du sens alors que l'ornement ne sert qu'à embellir'. Pourtant, en dehors du fait que les Grecs n'ont pas d'équivalent exact pour le mot «ornement ", les images des vases grecs montrent que des figures plus ou moins 
mimétiques interagissent avec des éléments stylisés et que ces derniers peuvent avoir une fonction figurative, voire sémantique ${ }^{10}$. Par conséquent, les "ornements» en abyme, sont certes un détail par rapport aux autres éléments de l'image, mais un détail qui n'est ni accessoire, ni insignifiant, ni gratuit. Au contraire, il est au service de l'ensemble : il incite le spectateur à se rapprocher de la surface du vase et à prendre plaisir au savoir-faire du peintre; il s'associe au luxe et au prestige des objets tout en révélant leur portée sémantique au sein de l'image; enfin, dans certains cas, le détail révèle des surprises et provoque l'étonnement ${ }^{11}$.

\section{Vêtements ${ }^{12}$}

4 Textes et images témoignent de l'importance du vêtement dans le système de valeurs grecques: marqueur d'identité et de comportement lié au sexe, à l'âge, à l'origine ethnique et au statut social, vecteur de messages politiques, économiques et religieux, signe de conformisme, de distinction ou d'altérité, parfois révélateur d'émotions, le vêtement est un vrai outil d'expression par sa forme, sa texture, sa couleur et son décor. Dans les épopées homériques, des habits et des étoffes fins, brillants et chatoyants, souvent confectionnés par les reines elles-mêmes, parent les palais ou sont offerts comme dons de prestige aux dieux et aux hôtes ${ }^{13}$. Le décor de ces œuvres royales est parfois rapporté de manière sommaire : Hélène tisse sur un manteau pourpre les épreuves que les Troyens et les Achéens ont subi à cause d'elle ${ }^{14}$. Ce décor autobiographique est une vraie mise en abyme: elle représente sur son ouvrage ce qu'Homère chante ${ }^{15}$. De son côté, Andromaque parsème sur le métier des "fleurs chatoyantes»(throna poikila) ${ }^{16}$. Ce dernier motif est repris par Sappho lorsqu'elle invoque Aphrodite comme poikilothronos, « aux multiples fleurs tissées ${ }^{17}$.

Figure 1

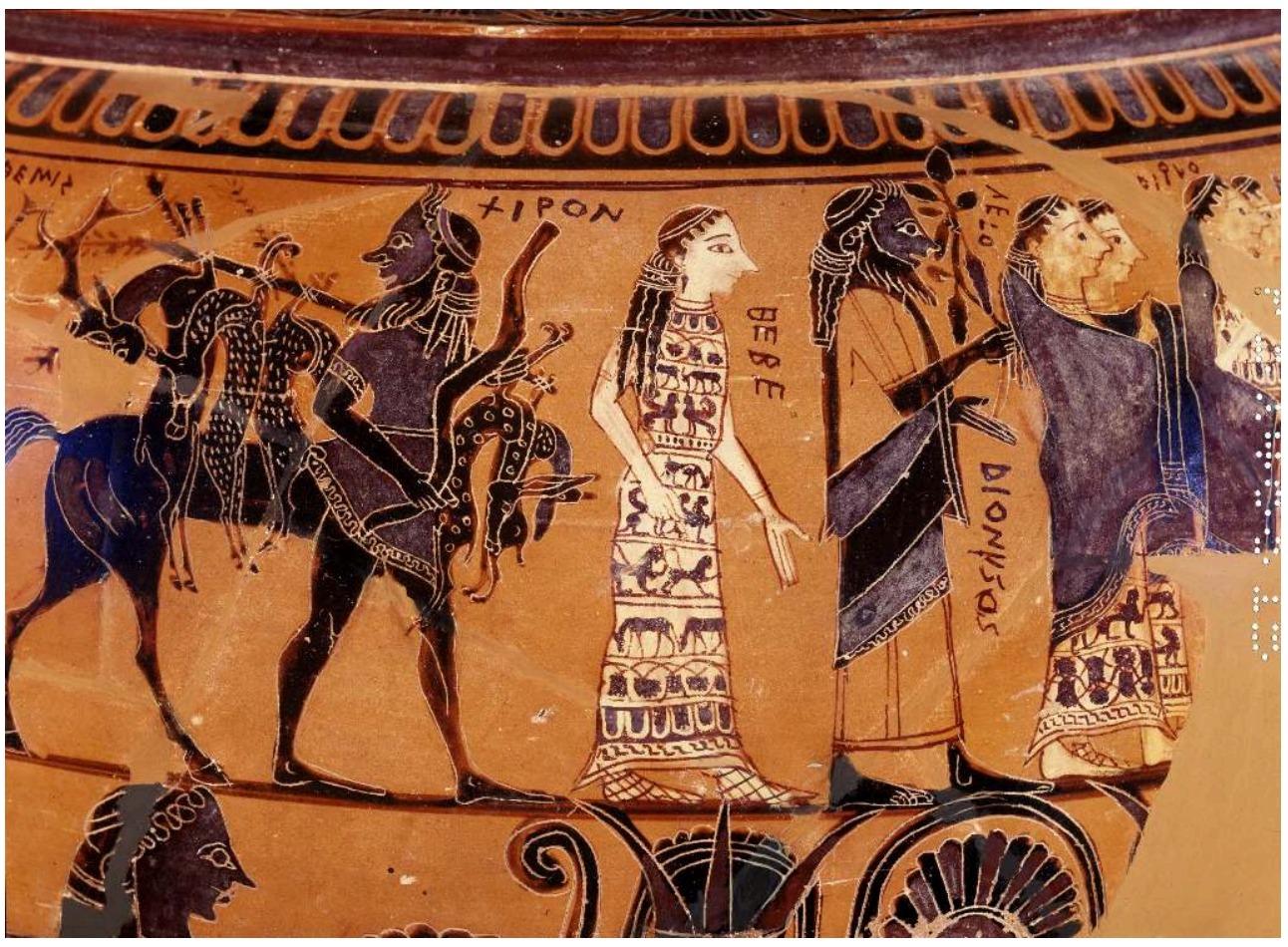

Dinos signé de Sophilos, 580-570, Londres, British Museum 1971.11-1.1. 
5 Alors que les vêtements fleuris abondent dans la céramique attique, rares sont ceux qui portent un décor plus complexe. Sur un dinos signé de Sophilos (580-570) ${ }^{\mathbf{1 8}}$, par exemple, la majorité des déesses participant aux noces de Pélée et de Thétis, portent des peploi blancs, divisés en plusieurs frises occupées par des motifs en silhouette : des chaînes florales (palmettes et «fleurs de lotus ») ${ }^{19}$, des animaux (cerfs, lions, panthères) et des sphinx: il s'agit de motifs orientalisants très fréquents dans la céramique grecque à partir du $\mathrm{VII}^{\mathrm{e}}$ siècle (fig. 1). La conception ornementale de ces peploi est identique à l'architecture picturale du vase, également divisée en frises contenant des motifs floraux, des animaux et des êtres hybrides. On en retrouve une variante dans la frise principale du fameux « vase François » signé du peintre Cleitias (vers 570) ${ }^{20}$, où le peplos noir d'une des Hôrai est orné de motifs floraux et de chars. Ces derniers, tirés par des chevaux ailés, sont une sorte de rappel micrographique des chars divins en route vers la demeure de Pélée et de Thétis, figurés dans la même frise ${ }^{21}$.

Figure 2

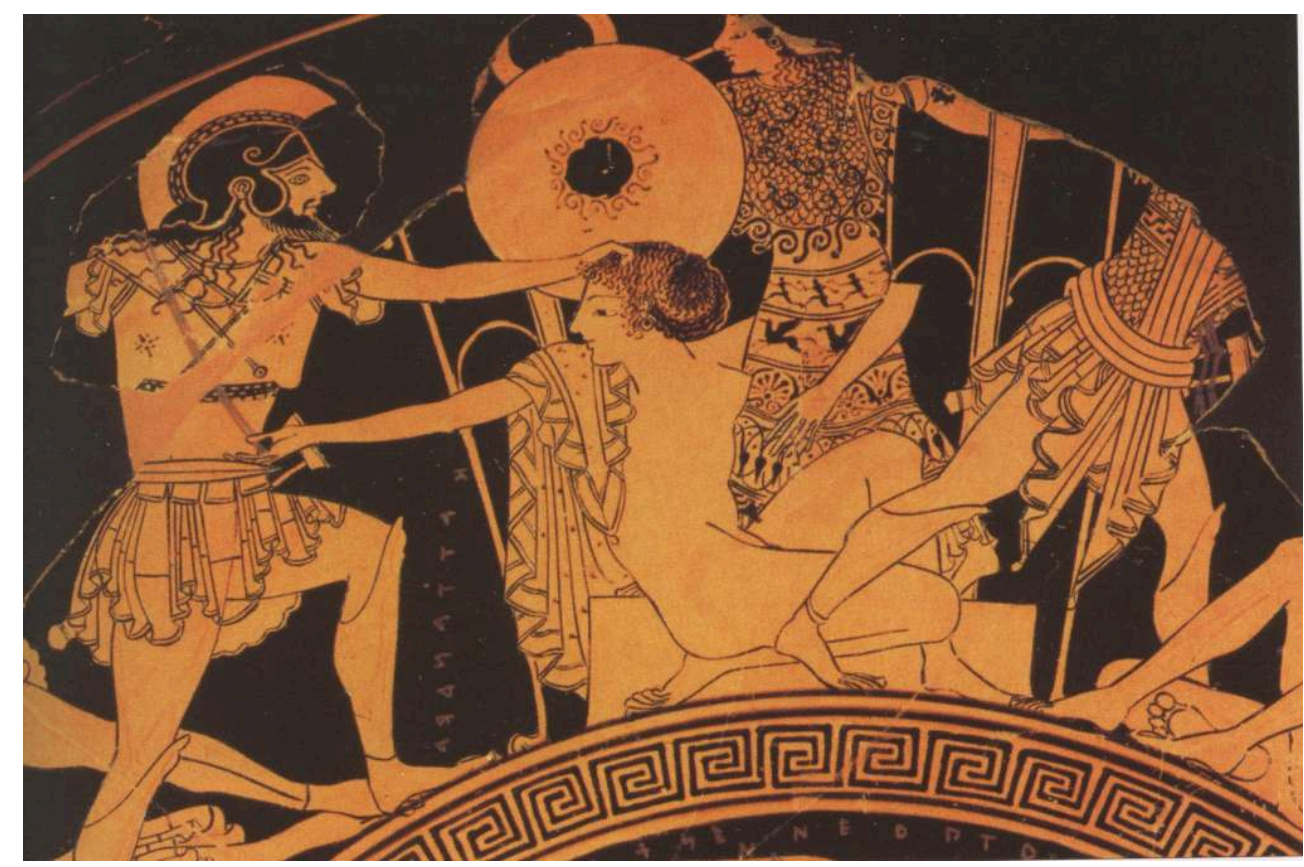

Coupe signée d'Euphronios, 500-490, Rome, Museo Nazionale Etrusco di Villa Giulia 121110. 
Figure 3

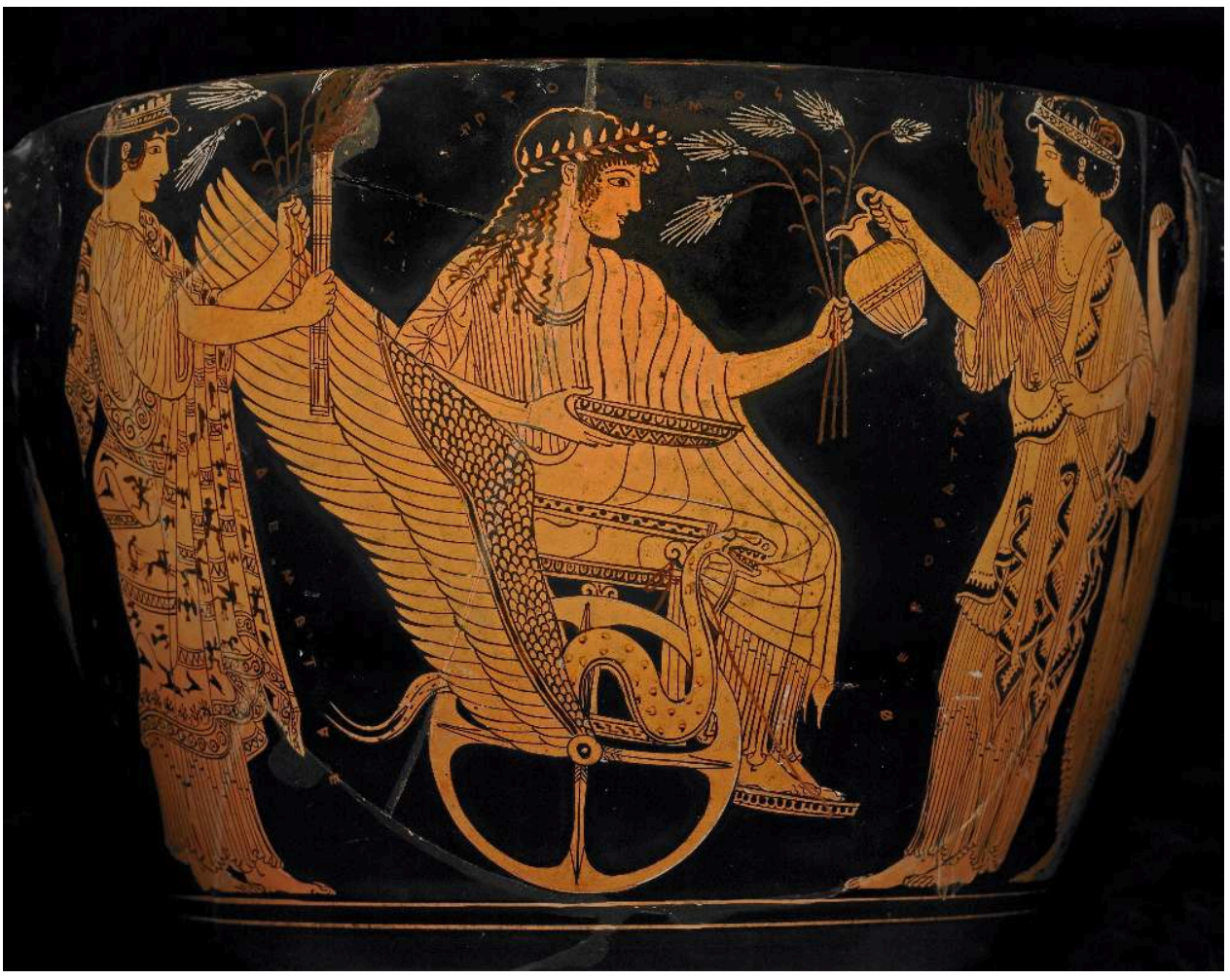

Skyphos attribué à Macron, vers 480, Londres, British Museum E140.

Figure 4

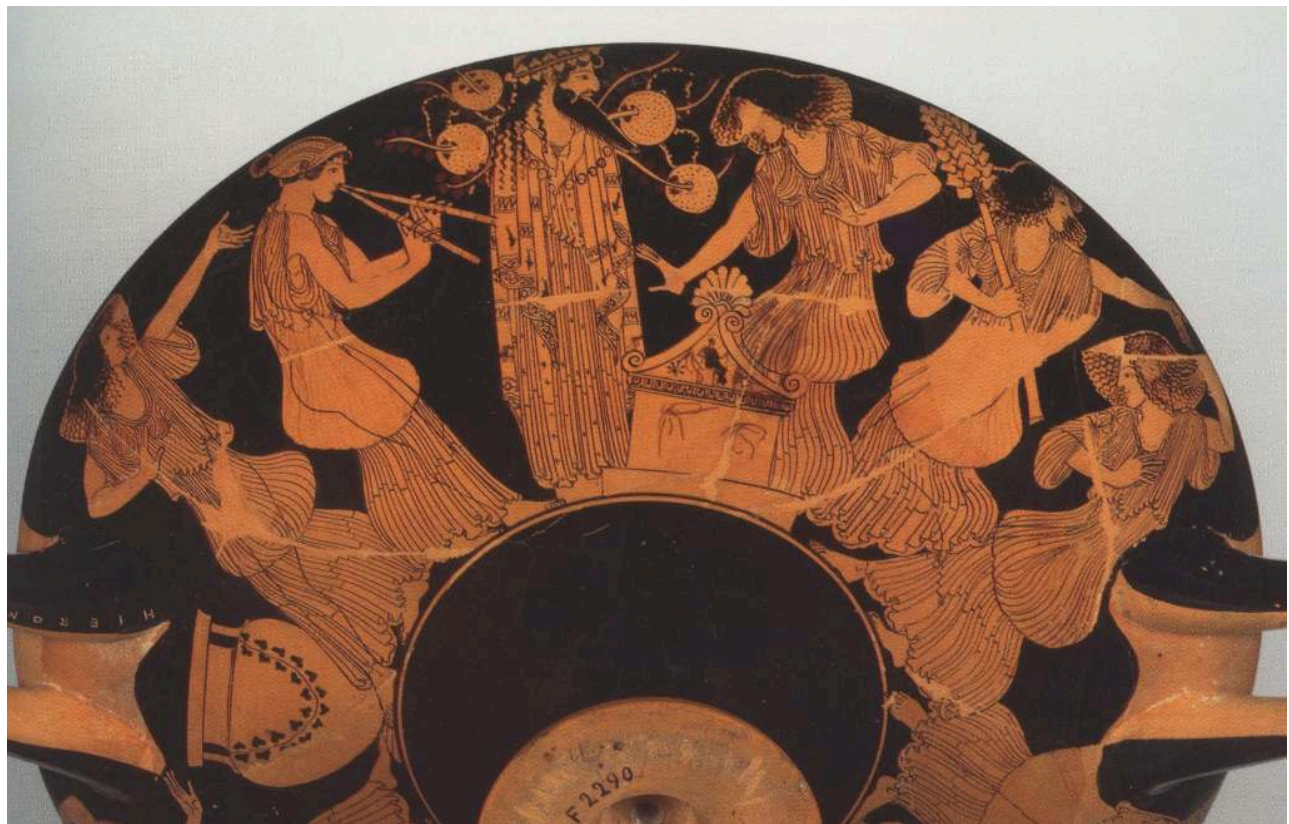

Coupe attribuée à Macron, vers 480, Berlin, Antikensammlung F2290.

6 Le vêtement divisé en frises survivra dans la céramique à figures rouges. Sur une coupe signée d'Euphronios (500-490) représentant l'Ilioupersis ${ }^{22}$, la statue d'Athéna porte un peplos au style "archaïsant »: des chaînes de fleurs de lotus et des chaînes de 
palmettes, telles qu'on les trouve respectivement sur les vases à figures noires et rouges, alternent avec des Pégases et des figures en train de courir (fig. 2). La richesse du décor contraste avec le corps dénudé de Cassandre, marque d'extrême vulnérabilité $^{23}$, alors que son caractère mouvementé rajoute à l'agitation et à la violence de la scène. L'idée de la vélocité liée à celle de la bigarrure sont de nouveau suggérées sur le skyphos de Macron à Londres ${ }^{24}$ (vers 480) aussi bien par le siège ailé de Triptolème (notons les écailles des ailes et la peau du serpent, éléments de poikilia) ${ }^{25}$ que par le manteau de Déméter : des frises au décor géométrique et floral (chaîne de palmettes) coexistent avec des frises occupées par des chars en course, des Pégases, des oiseaux, des figures ailées et des dauphins ailés (fig. 3). La présence des dauphins a une fonction supplémentaire, directement liée à l'usage du skyphos comme vase à boire : elle évoque l'imagerie maritime du symposion où l'andrôn est comparée à un navire, les convives aux rameurs de Dionysos, l'acte de boire ensemble à la traversée de la mer ${ }^{26}$. Le dauphin se trouve intimement lié à Dionysos aussi par le fait qu'il est l'agent de son action divine - rappelons que c'est en dauphin que le dieu a transformé les pirates tyrrhéniens qui ont voulu le kidnapper (Hymne homérique à Dionysos, VII). Pour toutes ces raisons, le dauphin devient, sur une autre coupe du même peintre à Berlin ${ }^{27}$, le motif principal de l'himation porté par l'idole de Dionysos: sa présence tout comme celle des femmes en frénésie (mania) occupant les deux côtés du vase, malgré la différence d'échelle, évoque l'effet de transformation que suscite le contact avec le dieu (fig. 4) ${ }^{28}$. Enfin, la disposition archaïque des motifs (dauphins et chars) à l'intérieur des frises souligne l'ancienneté de l'image du dieu.

Figure 5

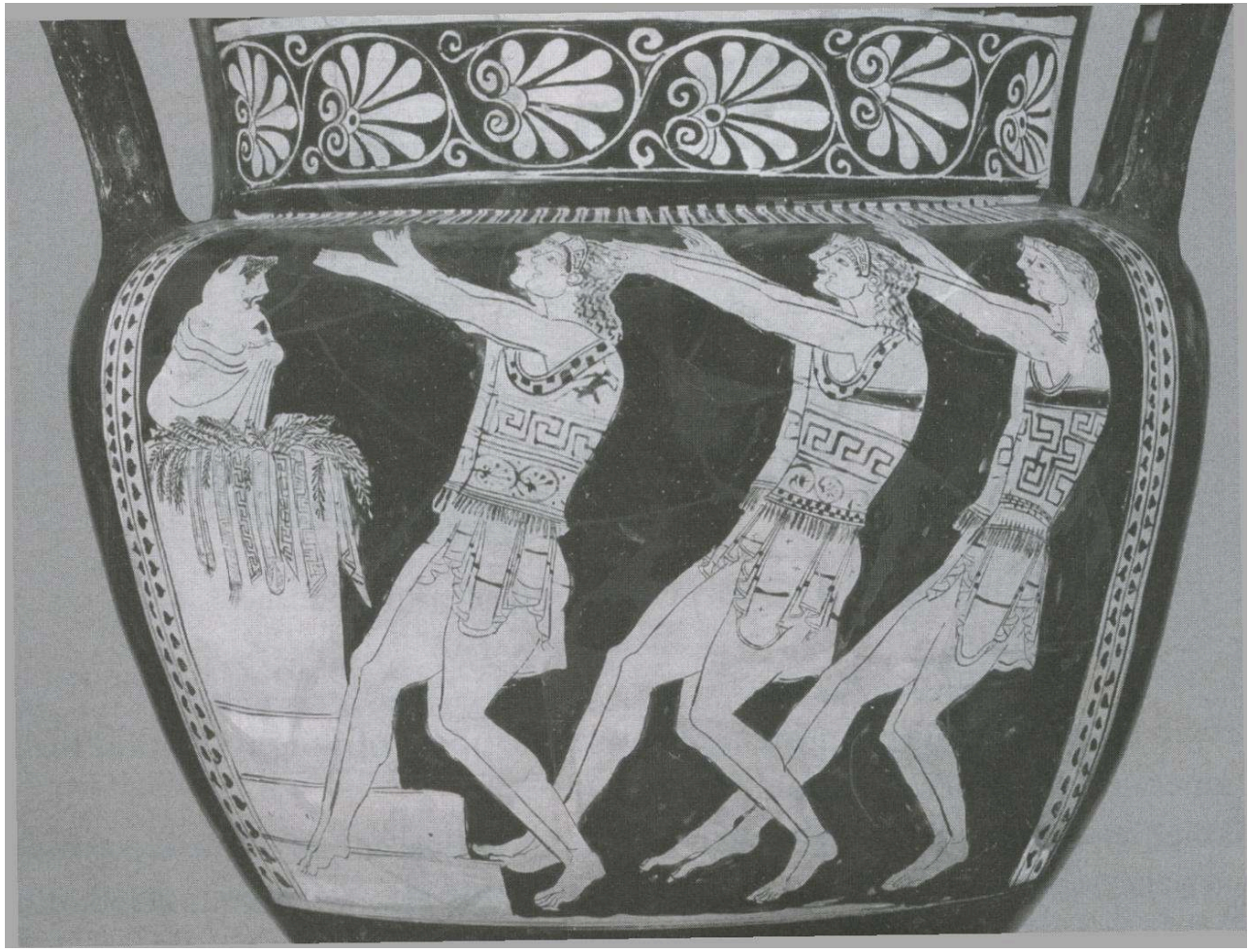

Cratère à colonnettes, vers 480, Bâle, Antikenmuseum und Sammlung Ludwig Bs415.

7 Les vêtements bigarrés ne sont pas l'apanage des dieux et de leurs statues. Un cratère à colonnettes (vers 480$)^{29}$ représente trois couples de jeunes hommes, peut-être des 
acteurs tragiques, exécutant une danse, les bras tendus et la bouche ouverte ${ }^{30}$, dans la direction d'une effigie ayant les traits d'un homme barbu, posée sur ou derrière une structure à degrés (fig. 5). Les costumes des danseurs sont ornés de palmettes et de méandres, motifs que l'on retrouve sur leurs stephanai ${ }^{31}$. De manière étonnante, sur le chiton porté par le premier danseur figure un satyre en train d'avancer, les jambes écartées, dans la même direction que le chœur, comme s'il participait lui aussi au spectacle de danse. Sa présence individualise le premier danseur tout en brisant la monotonie de la scène due à la répétition des gestes et des motifs vestimentaires. En même temps, elle renvoie au revers du vase où deux satyres s'agitent autour d'un cratère ${ }^{32}$.

Figure 6

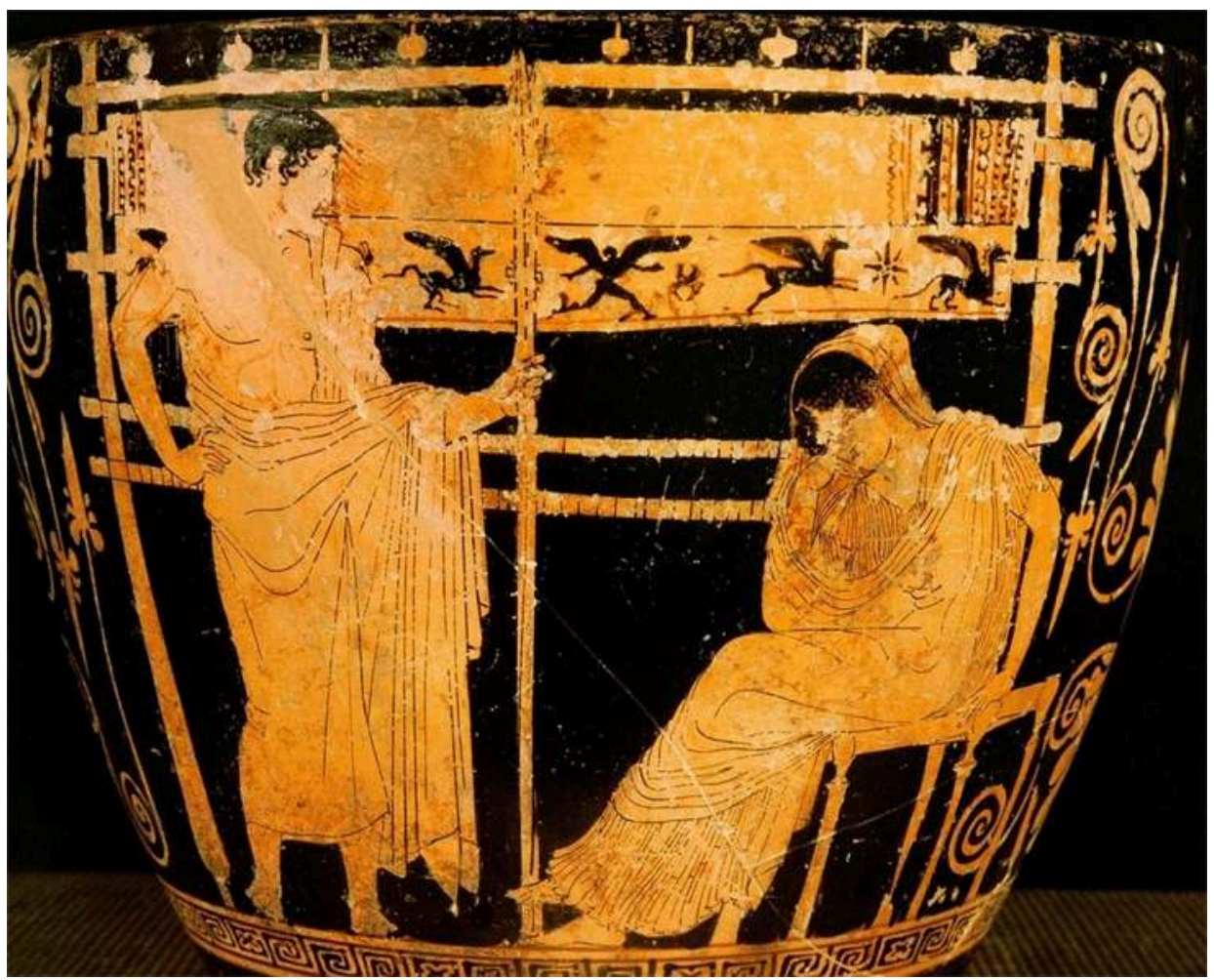

Skyphos attribué au Peintre de Pénélope, 450-440, Chiusi, Museo Archeologico Nazionale 1831.

Enfin, cas unique, le skyphos du Peintre de Pénélope (450-440) ${ }^{33}$ représente l'épouse d'Ulysse, accompagnée de son fils Télémaque, devant un grand métier à tisser dont la toile non achevée, est ornée d'une frise agitée, dans l'esprit "orientalisant»; on y distingue des hybrides ailés (notamment Pégase et un griffon) ainsi qu'un homme ailé (fig. 6) ${ }^{34}$. Leur mouvement de gauche à droite, soutenu par le regard de Télémaque, porte l'attention du spectateur sur le visage attristé de Pénélope. Une étoile rappelle qu'il s'agit d'« une pièce plus luisante que la lune et le soleil»(Odyssée, XXIV, 148) ${ }^{35}$. Alors qu'il n'est pas vraiment décrit par Homère, l'ouvrage devient ici le sujet principal de l'image, signe de la ruse (mêtis) de la reine, qualité qu'elle partage avec son mari figuré au revers du vase : tissé le jour, défait la nuit, il lui sert de prétexte pour retarder son choix parmi les prétendants ${ }^{36}$. Élément figuratif, signe et ornement, l'ouvrage bigarré déploie ici toutes les potentialités de son langage textile. 


\section{Armes}

Deuxième corps du guerrier ${ }^{37}$, les armes sont une source de fascination visuelle. D'abord, parce que la beauté et le chatoiement de leur surface soigneusement ouvragée répercutent le charme et l'excellence (aristeia) de leur propriétaire, sa charis héroïque ${ }^{38}$. Ensuite parce qu'ils suscitent l'effroi chez l'adversaire. En image, la beauté, l'éclat et le pouvoir de fascination des armes sont véhiculés par leur décor soigné, par leurs motifs stellaires, végétaux, animaliers ou autres. Notons qu'il s'agit des motifs que l'on retrouve sur des vraies armes (p.ex. les boucliers d'olympie), souvent combinés à ceux qui sont propres au répertoire ornemental des peintres attiques ${ }^{39}$. Incrustés ou incisés, ils assignent à la surface métallique de la splendeur et du mouvement, deux qualités englobées dans la notion d'aiolos, notion qui désigne tout ce qui est scintillant, sinueux, vif, mouvant ${ }^{40}$. Par leur surface composite réfléchissant un éclat coloré et miroitant, les armes rejoignent aussi les catégories de daidalon, de poikilon et d'agalma.

Parmi les pièces de panoplie, le bouclier est certainement la plus identitaire car l'épisème ${ }^{41}$ permet d'identifier son détenteur ${ }^{42}$. Dans l'Iliade, Homère fait une très longue description du décor qu'Héphaïstos applique sur le second bouclier d'Achille : animé et complexe, il représente tout un microcosme ${ }^{43}$. Dans les Sept contre Thèbes d'Eschyle, les épisèmes, semata, ornant les boucliers des sept chefs de guerre, possèdent une fonction prémonitoire ${ }^{44}$. Dans les deux cas, le décor des boucliers, loin d'être un ajout accessoire, est porteur d'un discours sémantiquement chargé ayant sa propre place dans le déroulement de l'intrigue. Il en va de même pour les images.

Figure 7

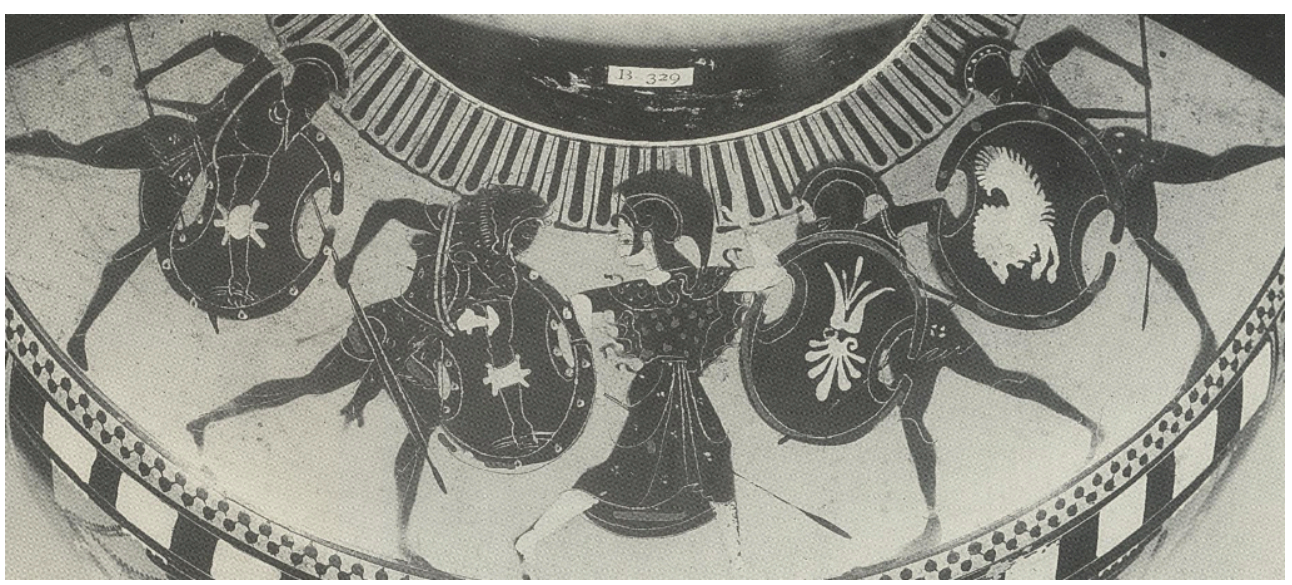

Hydrie attribuée au Peintre AD, 520-500, Londres, British Museum B329.

Le dossier des épisèmes étant largement étudié par François Lissarrague, je me limiterai à deux exemples peu connus. Le premier se trouve sur l'épaule d'une hydrie du Peintre AD (520-500) ${ }^{45}$, où l'un des guerriers porte un bouclier orné d'un foudre en forme de fleur, mi-palmette, mi-bouton (fig. 7). Le choix d'un tel motif ne doit pas surprendre : le foudre est avant tout l'arme redoutable de Zeus, à la fois insigne de suprématie et manifestation de colère divine. En tant qu'épisème, il signale la rapidité et l'impétuosité agressive du guerrier tout comme le lion ailé sur le bouclier de son adversaire à droite ${ }^{46}$ : les deux motifs transforment le bouclier, objet prophylactique, en arme offensive. Par un effet de synesthésie, le foudre signale aussi le vacarme des 
armes aussi terrifiant que le son du tonnerre. Sur le plan graphique, le blanc rappelle les épithètes de Zeus, aiolobrontês (maître de le foudre étincelante) ${ }^{47}$ et surtout argikeraunos (au foudre argent/blanc) ${ }^{48}$. En même temps, il visualise l'éclat aveuglant du foudre : condensé de feu et de lumière, il est décrit par Eschyle comme anthos pyros, le mot anthos ayant le double sens de «fleur» et d' "éclat " ${ }^{49}$. Cette métonymie trouve sa visualisation tout au long de la céramique attique où le foudre de Zeus adopte plusieurs formes florales : double bourgeon, double «fleur de lotus » ou bourgeon et «fleur de lotus $\aleph^{50}$. Rien d'étonnant, alors, dans le fait que les deux motifs, la fleur et le foudre, ayant comme point commun la luminosité ardente, se combinent pour former l'épisème d'un bouclier.

Figure 8

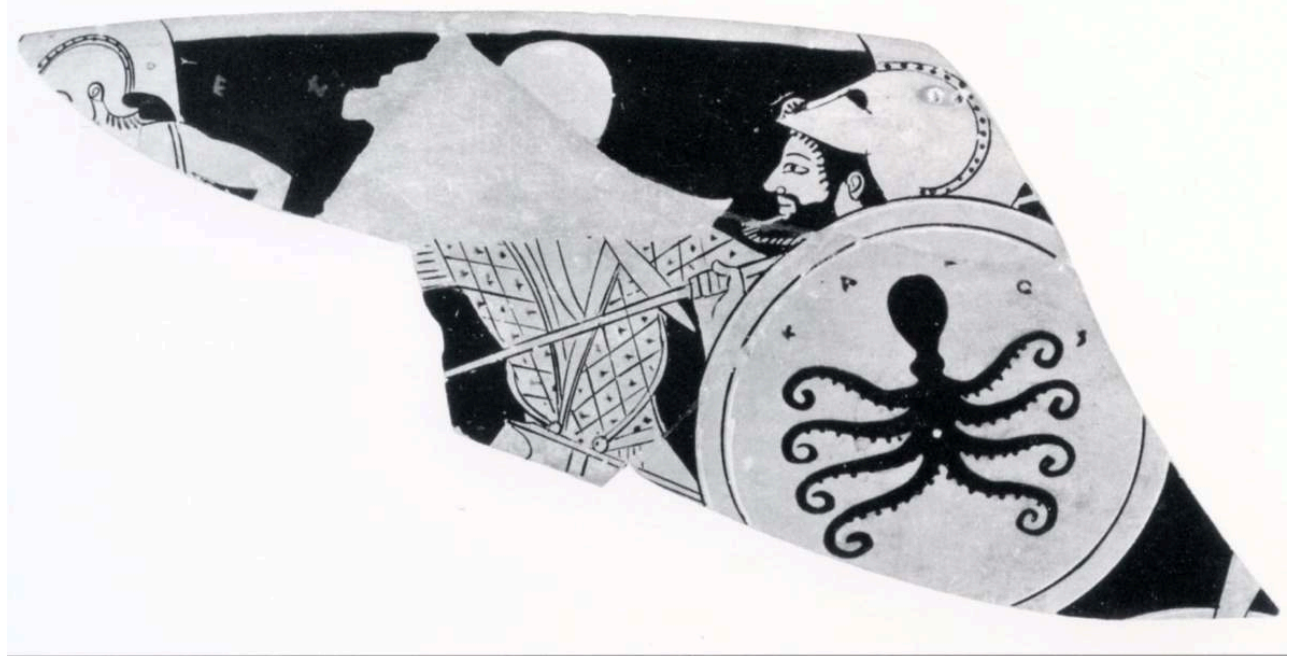

Fragment d'une coupe attribuée à Epictétos, 510-500, Malibu, J. P. Getty Museum 86.AE.306.1-4.

Le second exemple est le fragment d'une coupe d'Epictétos (510-500) ${ }^{51}$ où le bouclier d'un hoplite est orné d'un poulpe (fig. 8). Le choix n'est pas dû au hasard : polyplokos, aux nombreux replis, et dolomêtis, fourbe, le poulpe est capable de s'adapter au corps qu'il saisit, par sa forme et par sa couleur ${ }^{52}$. À l'instar du poulpe, un bon guerrier doit impérativement faire preuve de mêtis, notion évoquant la ruse, la promptitude, la duperie. Figuré de face et nimbé par l'inscription laudative «kalos », le poulpe énonce ici les qualités du guerrier tout en suscitant un effet de fascination apotropaïque sur l'adversaire ${ }^{53}$. 


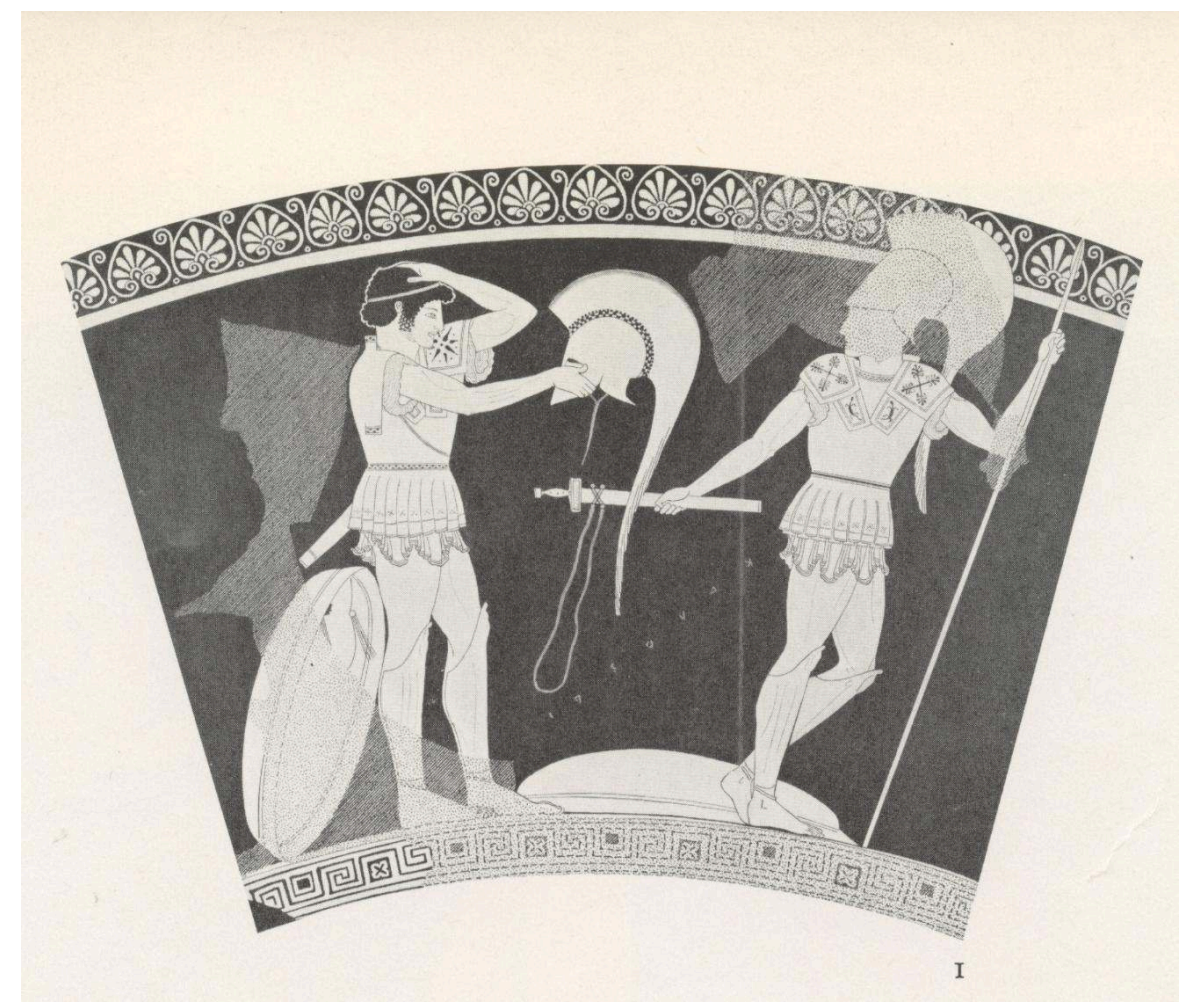

Cratère attribué au Peintre de Cléophradès, vers 490, New York, Metropolitan Museum of Art 08.258.58.

Autres éléments de protection, la cuirasse et le casque peuvent recevoir des motifs semblables à ceux des boucliers. Ainsi, sur un cratère du Peintre de Cléophradès (vers $490)^{54}$, les cuirasses des deux guerriers, est ornée d'étoiles, de palmettes et de deux lézards sur les épaulières (pteryges) (fig. 9). La présence des motifs stellaires, que l'on retrouve sur les boucliers, rappelle l'éclat du bronze; pas étonnant qu'Homère compare la cuirasse de Patrocle à un ciel constellé d'étoiles (Iliade, XVI, 133-4). L'éclat et la finesse du travail sur le bronze, sont aussi visualisés par les palmettes. Toutefois, la présence du lézard est plus difficile à cerner : animal souvent représenté dans des scènes où figurent des chars, scènes de départ du guerrier et scènes de bataille, il semble communiquer l'idée de la vélocité, une des qualités principales du guerrier ${ }^{55}$. 


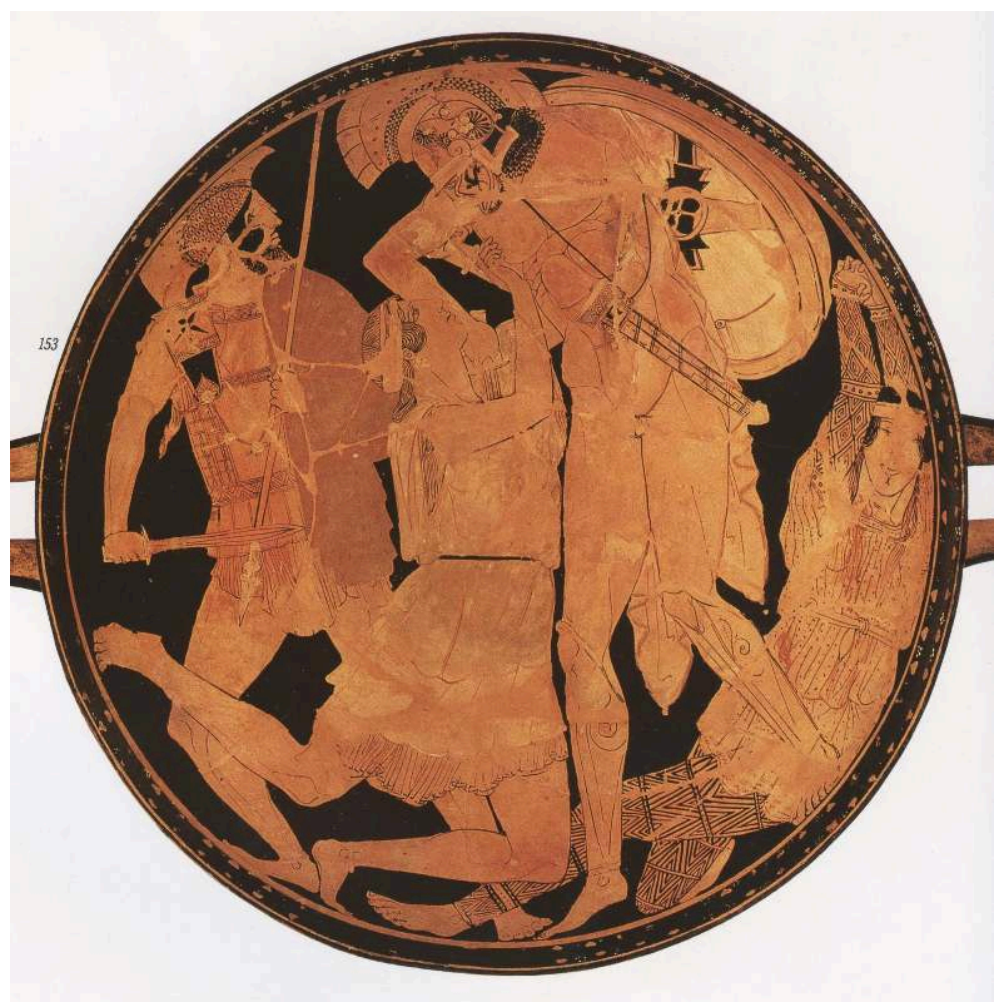

Coupe attribuée au Peintre de Penthésilée, 460-450, Munich, Antikensammlungen 2688.

Le casque devient un élément figuratif à part entière, à l'intérieur d'une coupe du Peintre de Penthésilée (460-450) ${ }^{56}$, où Achille s'apprête à tuer la belle Amazone (fig. 10). Sur la calotte ornée d'une composition florale à trois palmettes, un bouton de fleur, mis en relief grâce à l'usage de la barbotine dorée, et secondé par un sphinx ornant le parejoue, oriente l'attention du spectateur vers le regard échangé entre les deux figures, regard qui, selon la tradition écrite, suscitera chez Achille une passion amoureuse pour sa victime mourante ${ }^{57}$. Autrement dit, le décor s'anime pour soutenir et transmettre aux spectateurs le récit figuratif de la manière la plus explicite et la plus visuellement efficace.

\section{Meubles de luxe}

Dans l'économie anthropocentrique des vases attiques, la majorité des meubles fonctionne comme une sorte de prolongement du corps humain ou divin : le trône, les différents types de sièges ainsi que le lit de banquet révèlent le statut, l'importance et le rôle de la personne qui les occupe, et en ce sens, ils participent activement au contenu figuratif de l'image. Leur décor, malgré son caractère plutôt conventionnel et standardisé, leur octroie à la fois un aspect luxueux et une prégnance sémantique ${ }^{58}$.

D'abord, le trône : chef d'œuvre d'ébénisterie, il fait partie de ces objets qualifiés de daidalea ou poikila. Lorsque Thétis arrive chez Héphaïstos, Charis, l'épouse du dieu, l'invite à s'asseoir «sur un siège à clous d'argent, un beau siège ouvragé (thronos argyroèlos, kalos daidaleos) avec un banc sous les pieds ${ }^{59}$. La présence de Charis, incarnation de la grâce physique, ajoute à l'idée de la pulsation visuelle émanant des 
œuvres de son époux. La description du trône de Zeus à Olympie, rapportée par Pausanias, véhicule cette même idée de vibration lumineuse issue de l'assemblage des matériaux : «Le trône est incrusté (poikilos) d'or et de pierres et aussi incrusté (poikilos) d'ébène et d'ivoire et on y a peint des animaux qui sont entremêlés et on y a sculpté des agalmata... $»^{60}$. En image, ce sont les différents motifs, végétaux et animaliers, qui évoquent l'alliage de différents matériaux, (or, argent, ivoire et pierres précieuses), la polychromie et la luminosité du trône. Ce faisant, ils mettent en exergue la beauté, l'éclat et le pouvoir de la personne qui trône, dieu ou roi.

Figure 11

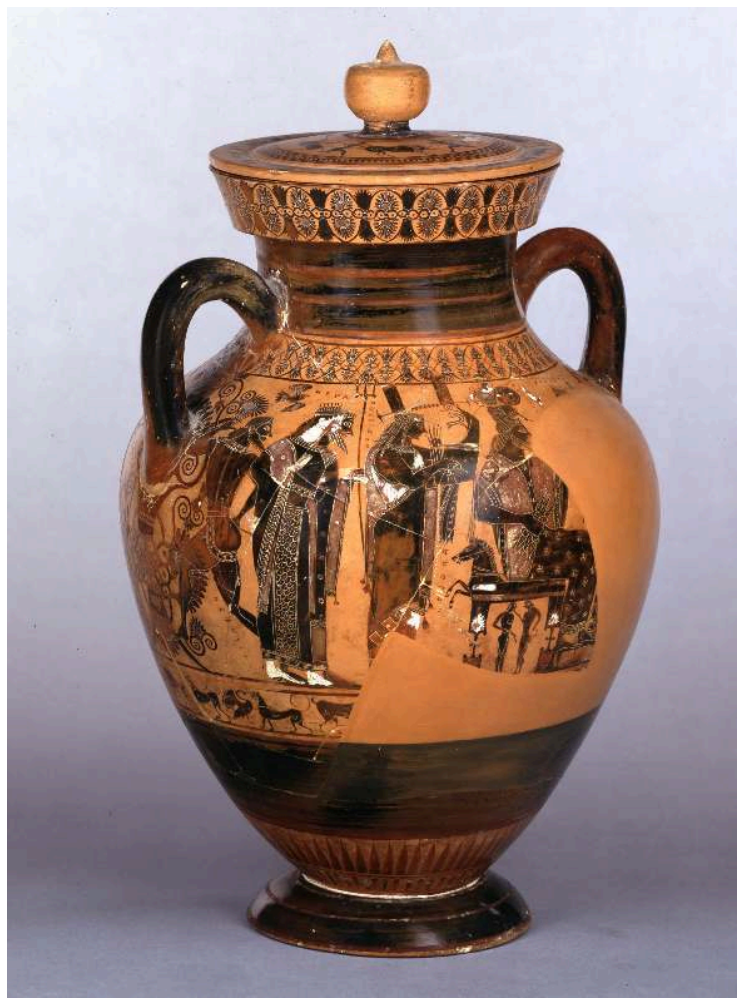

Amphore attribuée au Groupe E, 550-540, Londres, British Museum B147

17 Les Peintres du Groupe E excellent à la représentation du trône de Zeus, produit de l'ingéniosité d'Héphaïstos, une vraie merveille pour les yeux ${ }^{61}$. Sur une amphore représentant la naissance d'Athéna $(550-540)^{62}$, le dossier raccourci du trône prend la forme de l'avant-train d'un cheval; sa présence évoque la scène du revers où un guerrier s'apprête à monter sur un char tiré par des chevaux mais aussi la scène du couvercle où des cavaliers s'adonnent à la chasse (fig. 11). Sous le siège figure un couple de jeunes hommes nus, dont la petite échelle contrebalance celle d'Athéna sortie de la tête de Zeus. Il y a là une vraie mise en abyme : encadrés par le décor floral des pieds du trône, les deux hommes, l'un d'eux tenant une couronne, forment une saynète de cour homoérotique, sujet très fréquent dans la céramique attique contemporaine. Sur d'autres vases à figures noires, le décor plastique sous le siège peut prendre la forme d'un ou deux lions ${ }^{63}$, d'un cygne ${ }^{64}$, d'un homme drapé $e^{65}$, d'un éphèbe nu et accroupi ${ }^{66}$, d'une figure ailée (Iris ? ${ }^{67}$, d'une sirène $e^{68}$, d'une sphinx ${ }^{69}$ ou d'un démon ailé à tête de dauphin ${ }^{70}$. Il est intéressant de noter que ce type de trône apparaît presque exclusivement dans les scènes de naissance d'Athéna; dans ce contexte le trône de Zeus 
au décor animé, se présente comme un objet surnaturel, comme un thauma à l'instar de la naissance miraculeuse de la déesse.

Figure 12

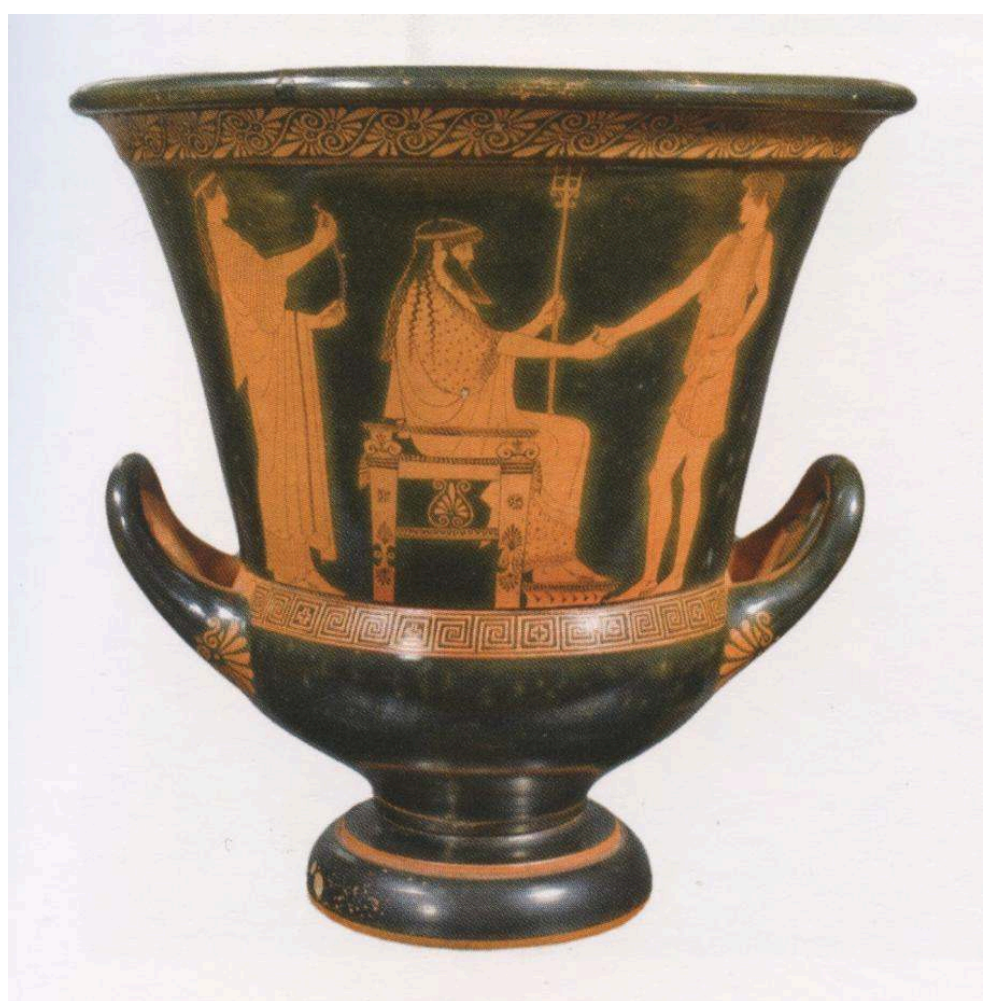

Cratère en calice attribué au Peintre de Syriskos, 480-470, Paris, Cabinet des Médailles 418

Sur un cratère du Peintre de Syriskos (480-470) $)^{71}$, c'est un autre thauma qui est suggéré, celui du plongeon de Thésée dans le royaume maritime de Poséidon, son père divin, afin de récupérer l'anneau en or de $\operatorname{Minos}^{72}$ : le jeune héros, debout, esquisse un geste d'alliance avec le dieu trônant, en présence d'Amphitrite (fig. 12). Alors que le trône est orné de nombreuses palmettes en écho avec celles du décor du vase, le petit support sur lequel Poséidon pose ses pieds est orné de dauphins : ceux-ci dénotent le royaume maritime où se déroule la rencontre entre le dieu et le jeune héros, leur image micrographique étant une "mer implicite $»^{73}$; en même temps, ils rappellent que ce sont eux qui ont conduit Thésée vers la demeure de son père ${ }^{74}$. Pour le dire autrement, le décor du repose-pied sert à la fois d'indicateur spatial mais aussi de micro-récit rappelant une séquence antérieure à la scène, celle du voyage de Thésée ${ }^{75}$. 
Figure 13

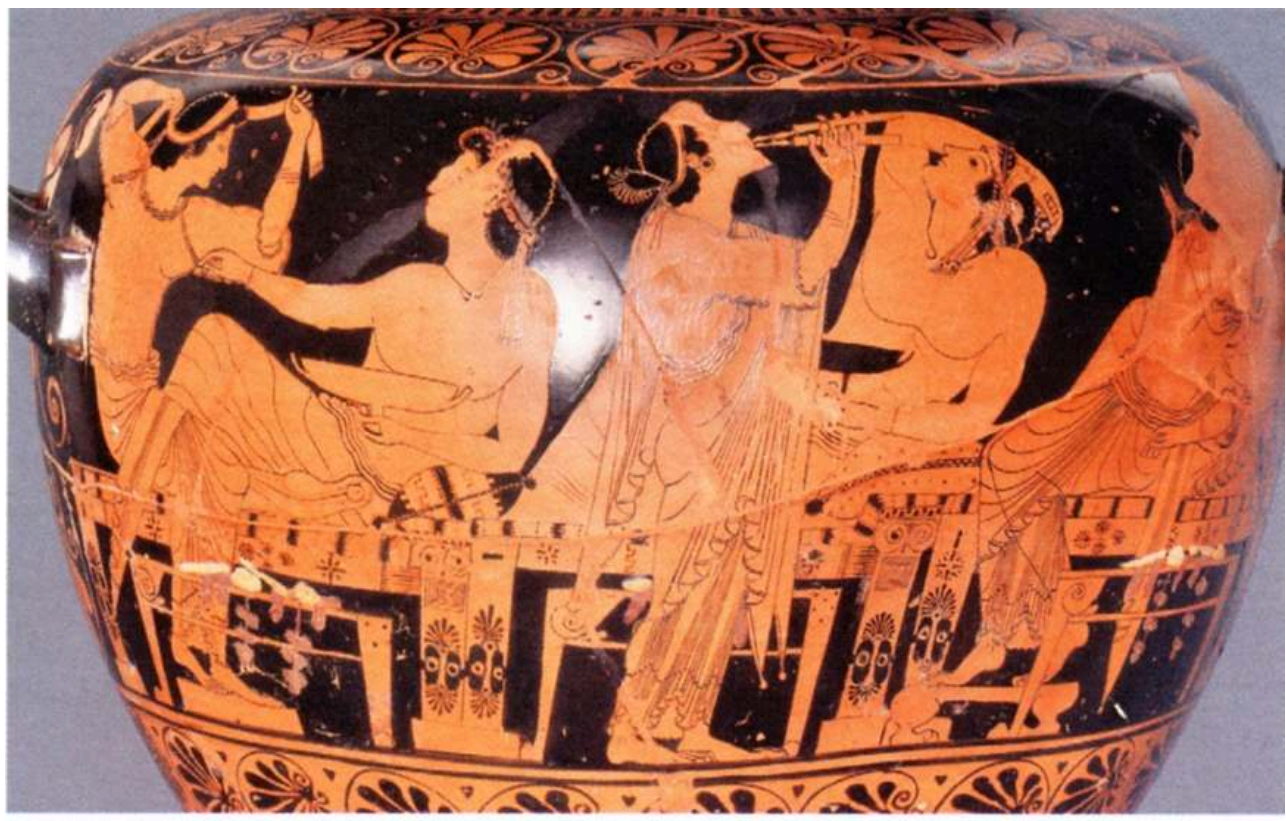

Stamnos signé par le peintre Smicros, 510-500, Bruxelles, Musées Royaux A717.

Le lit (klinê), composante essentielle du symposion, est sans doute le meuble le plus fréquemment figuré sur les vases attiques ${ }^{76}$. Issu de l'assemblage de matériaux divers, ${ }^{77}$ couvert de beaux tissus bigarrés et porteur d'un riche décor (palmettes, rosaces, motifs stellaires et motifs animaliers), il participe à l'atmosphère d'opulence, de bien-être et d'allégresse du banquet. Le stamnos signé par le peintre Smicros (510-500) ${ }^{78}$ en est sans doute le plus bel exemple : les demi-, les double- ou les croix de palmettes sur les lits répondent aux palmettes du cadre de l'image, renforçant l'idée de la charis dans toutes ses déclinaisons : beauté, abondance, échange, joie, plaisir (fig. 13). 
Figure 14

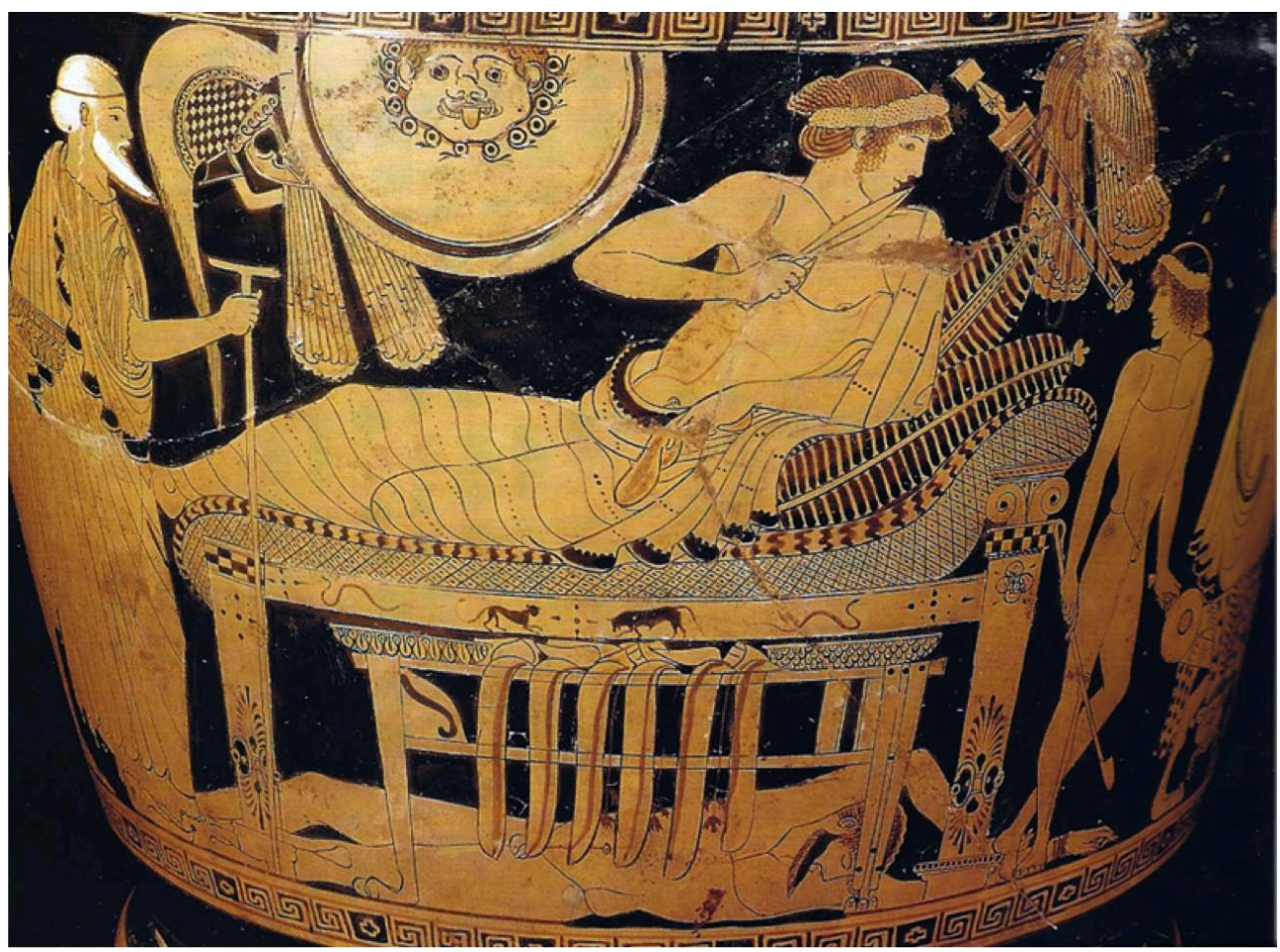

Skyphos attribué au Peintre de Brygos, vers 480, Vienne, Kunsthistorisches Museum 328.

Or, un lit diffère de tous les autres lits de banquet: il s'agit de celui d'Achille dans les scènes de la "Rançon de Priam $»^{79}$. Sur le célèbre skyphos du Peintre de Brygos (vers $480)^{80}$, Achille est allongé sur un lit en dessous duquel se trouve le cadavre dépouillé et outragé d'Hector (fig. 14). Le lit de banquet prend, de manière blasphématoire, la place de sa tombe. Son riche décor souligne davantage l'hybris d'Achille représenté en train de festoyer, un couteau et une tranche de viande dans chaque main. Parmi les divers motifs ornant le lit, certains sont de vrais pictogrammes qui éclairent l'arrière-plan de cette image. C'est le cas des deux serpents, animaux à la fois dangereux et funestes ${ }^{81}$, et surtout de la panthère ${ }^{82}$ et du taureau ${ }^{83}$ dont le face-à-face rappelle celui d'Achille et d'Hector ${ }^{84}$. 
Figure 15

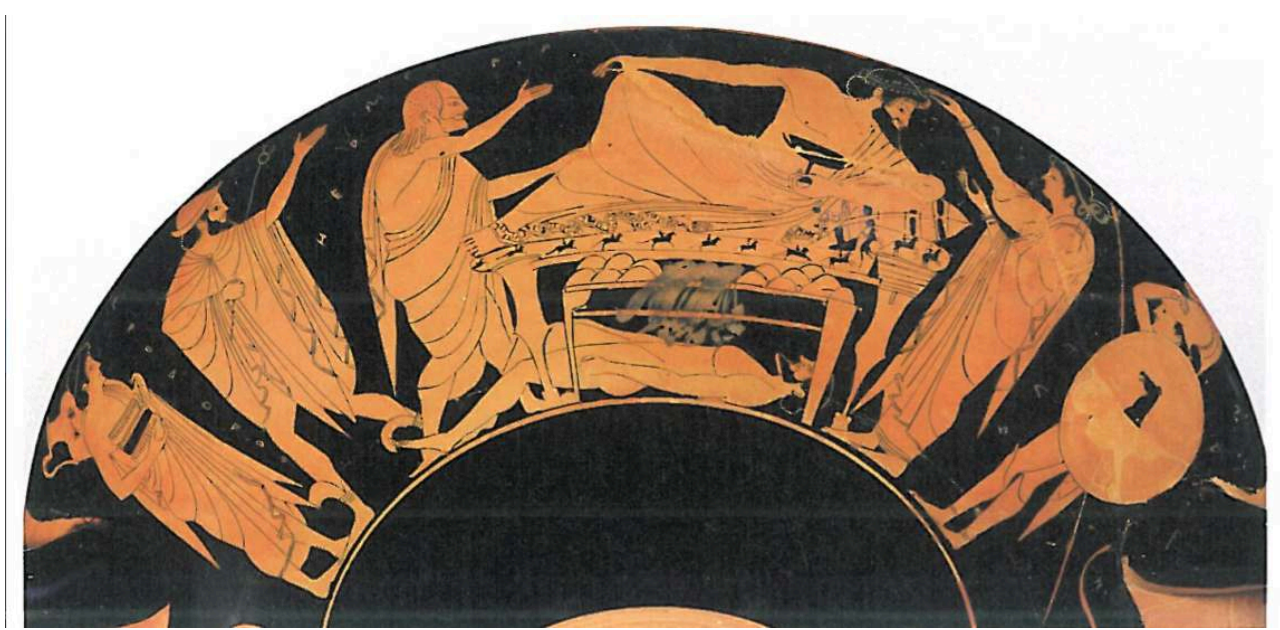

Coupe attribuée à Oltos, 520-510, Munich, Antikensammlungen 2618.

Sur une coupe d'Oltos (520-510) représentant le même sujet ${ }^{85}$, ce n'est plus le lit mais le matelas qui porte un décor tout à fait exceptionnel: il s'agit d'une longue série de cavaliers au galop (fig. 15). Cette image mouvementée, qui n'est pas sans rappeler celles des tissus vus plus haut, opère sur plusieurs niveaux : elle renforce l'inertie du cadavre d'Hector étendu à terre, elle fait écho à la tête de cheval qui sert d'épisème au bouclier de l'hoplite assistant à la scène, et enfin évoque l'image du revers où trois chevaux sont solennellement conduits par de jeunes serviteurs afin d'être présentés comme cadeaux à Achille. Il est évident que le choix du décor d'un élément aussi secondaire que le matelas d'un lit, n'est pas gratuit mais renforce le sens de l'image.

\section{Vases}

Le jeu de réflexivité ou de réitération qui consiste à représenter des vases céramiques sur des vases, a été minutieusement analysé par de nombreux spécialistes qui ont tous souligné son importance sémantique ${ }^{86}$; car non seulement il densifie le sens de l'image, mais il permet aussi d'entrevoir une certaine fierté professionnelle de la part des potiers et des peintres vis-à-vis de leurs propres productions. 
Figure 16a

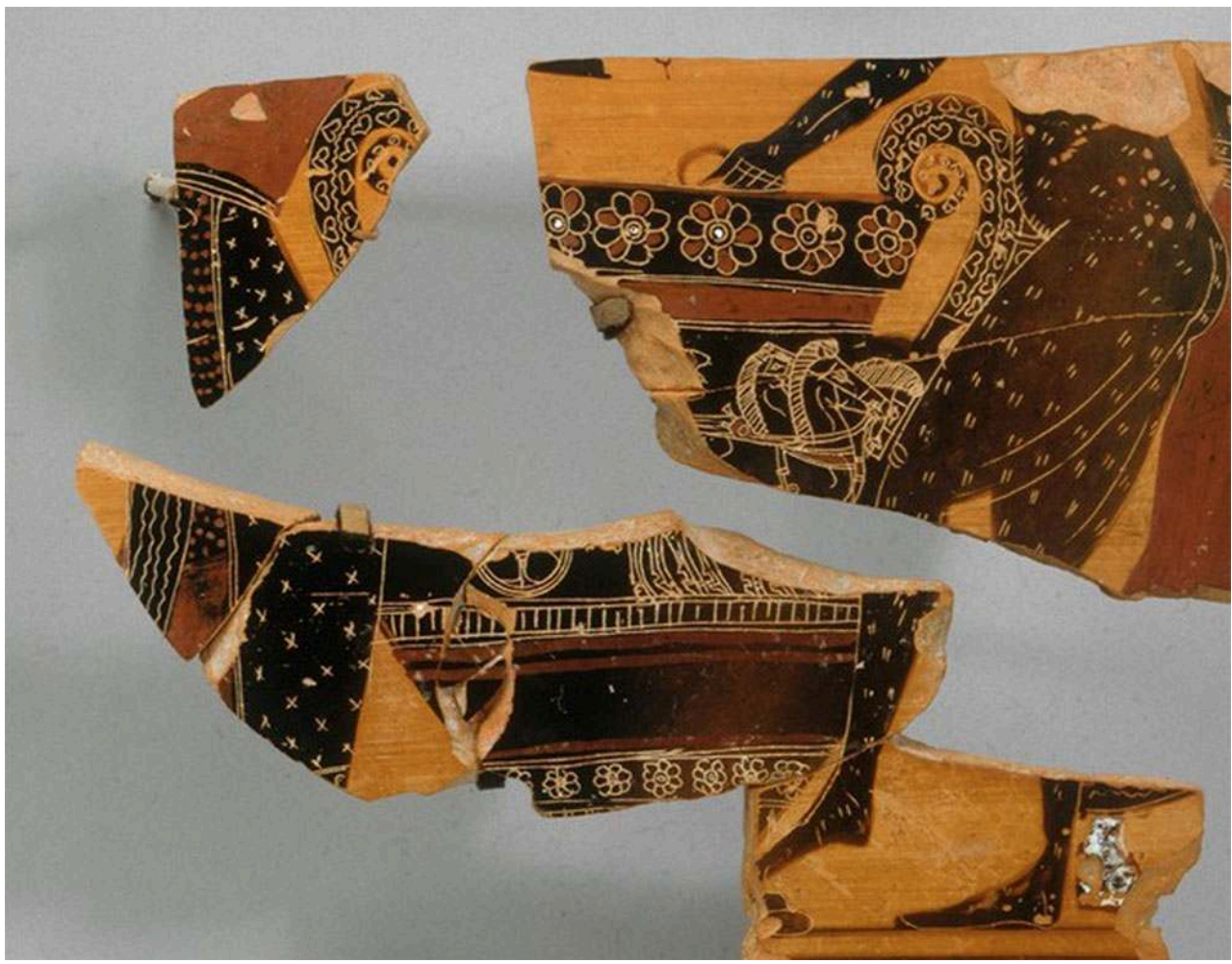

Fragments d'un cratère à colonnettes attribué à Lydos, vers 560, New York, Metropolitan Museum of Art 1997.388a-eee; 1997.493; 1996.56a. 
Figure 16b

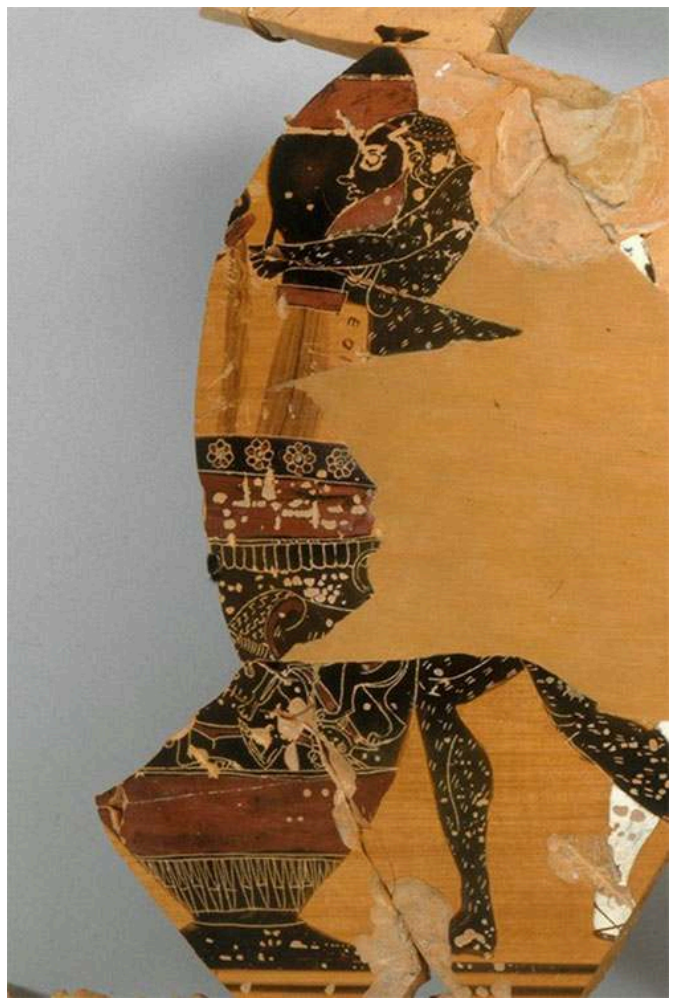

Fragments d'un cratère à colonnettes attribué à Lydos, vers 560, New York, Metropolitan Museum of Art 1997.388a-eee; 1997.493; 1996.56a.

Un des exemples les plus anciens est un cratère à colonnettes, malheureusement très fragmentaire, attribué à Lydos (vers 560$)^{87}$ : on y voit des satyres et des nymphes accompagnant Héphaïstos lors de son retour sur l'olympe. De manière exceptionnelle, le cratère met en scène au moins sept vases en terre cuite. Deux cas vont retenir notre attention : sous une anse, un satyre plonge son récipient (œnochoé) à l'intérieur d'un cratère à volutes (fig. 16a). Sous l'autre anse, un satyre et une nymphe (?) versent du vin à l'intérieur d'un second cratère (à volutes ou à colonnettes) (fig. 16b). La panse du premier cratère est ornée d'un char tiré par quatre chevaux; des feuilles de lierre, plante dionysiaque par excellence, ornent les anses alors que des rosaces polychromes parcourent la lèvre et la partie inférieure de la panse ${ }^{88}$. Une chaîne de rosaces orne également la lèvre du second cratère dont la panse représente un lion en train d'attaquer un taureau tombé à terre. On constate que Lydos prête beaucoup d'attention au décor secondaire des deux cratères, explicitement présentés comme des cratères en terre-cuite, ainsi qu'à leurs scènes en miniature (le char et le combat des animaux) véhiculant ici comme dans les exemples précédents, des connotations héroïques ${ }^{89}$. 
Figure 17

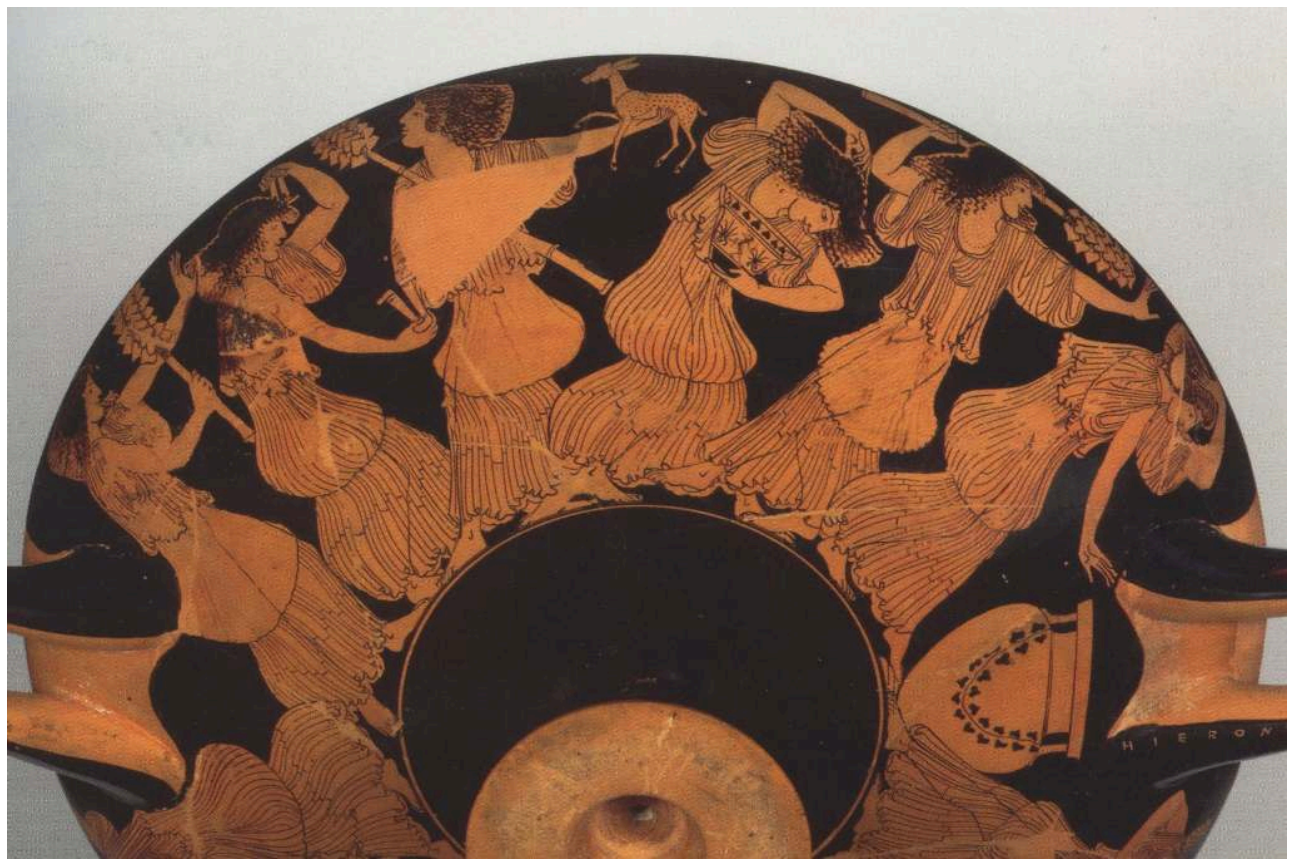

Coupe attribuée à Macron, vers 480, Berlin, Antikensammlung F2290. et sémantiques de la mise en abyme à trois reprises: d'abord, par le vêtement archaïque de l'effigie dont le décor renforce le caractère dionysiaque et agité de la scène. Puis, par l'image en silhouette du skyphos tenu par une des femmes: on y distingue entre deux palmettes, un satyre, « contrepoint masculin du cortège féminin de Dionysos $»^{90}$, dont la présence miniaturisée infuse une note de lubricité au sein du thiase (fig. 17). Le décor du skyphos répond en quelque sorte à l'image peinte sur le fronton de l'autel où l'on voit entre deux palmettes, une figure assise près d'un pied de vigne, sans doute Dionysos. L'image peinte, la palmette sculptée qui couronne le fronton, mais aussi les rehauts rouges évoquant des traces de sang, révèlent la surface de l'autel qui capte, ainsi, par son aspect fort élaboré, l'attention du spectateur. L'autel, l'effigie et le skyphos, tous les trois supports d'une imagerie dionysiaque, sont des agréments importants, même indispensables, au bon déroulement de la fête dionysiaque. 


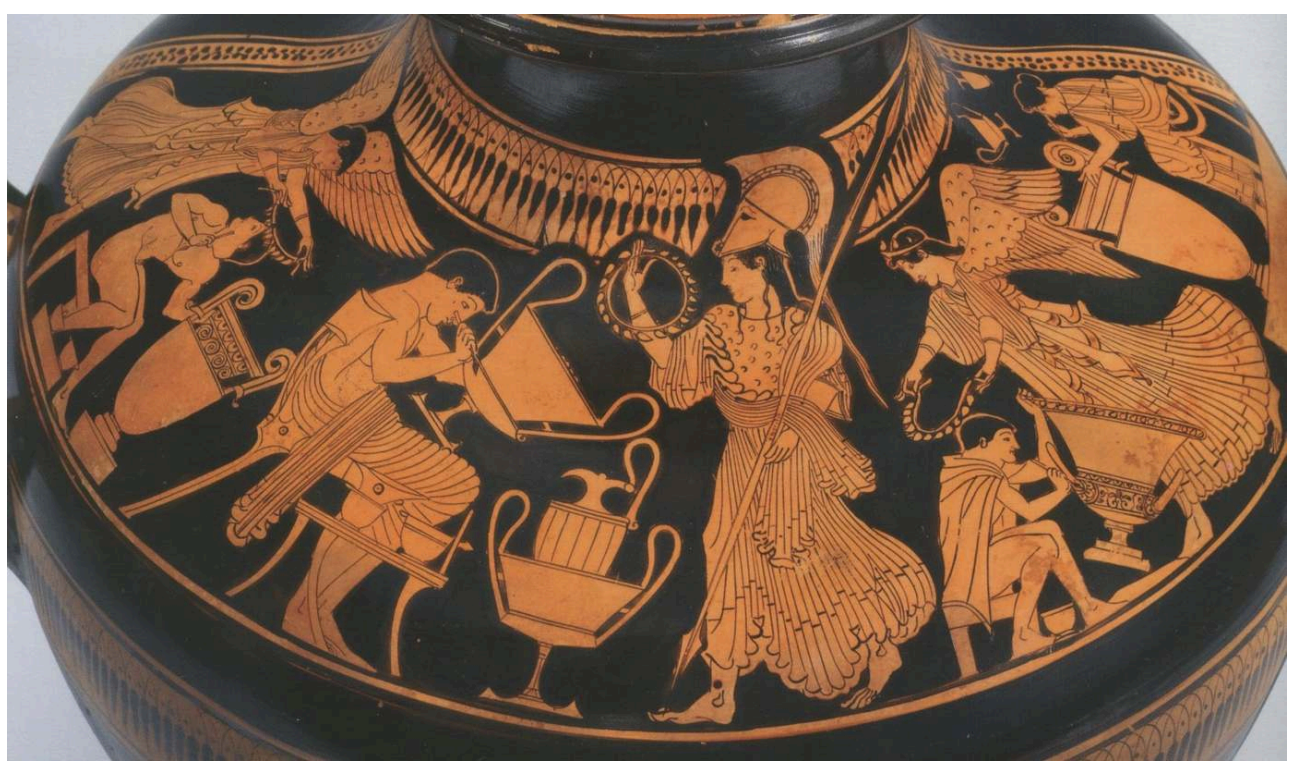

Hydrie attribuée au Peintre de Leningrad, 470-460, Vicence, Palazzo Leoni Montanari.

Dernier exemple, la fameuse hydrie du Peintre de Leningrad (470-460) ${ }^{91}$ représentant sur son épaule un atelier de potiers recevant des honneurs divins: Athéna et deux Nikès viennent couronner trois artisans mais semblent avoir oublié la jeune femme qui, figurée tout à droite, décore un cratère à volutes (fig. 18). L'artisan du centre travaille sur un canthare de taille monumentale alors que les deux autres réalisent le décor de leurs cratères: oves et spirales pour le premier, oves, palmette et feuilles pour le deuxième. Il est intéressant de noter que ces artisans sont en train de peindre non pas les belles scènes ornant souvent les cratères mais leur décor secondaire. Quoi qu'il en soit, l'image célèbre le savoir-faire du peintre, la maîtrise de ses gestes techniques, ainsi que ses productions à la taille monumentale et au décor soigné. Ce dernier octroie aux vases céramiques une poikilia qui, épaulée par l'éclat de la surface polie et lustrée, fait oublier leur matière humble. Car, tout comme les vases en métal, les vases en terre cuite ont été occasionnellement désignés comme des agalmata ${ }^{92}$.

\section{Monuments funéraires}

Il est connu que les peintres athéniens sont économes en ce qui concerne la représentation spatiale : un autel ou une stèle funéraire seuls, par exemple, suffisent pour désigner respectivement deux types d'espace sacré, l'un comme lieu d'échange privilégié avec les dieux, l'autre avec les morts. Constructeurs d'espace ${ }^{93}$, l'autel et la stèle jouissent souvent d'une place centrale au sein de l'image ainsi que d'un traitement graphique mettant en relief toute leur portée sémantique. Il faut ici noter qu'en dehors de leur décor sculpté ou peint, ils avaient droit à d'autres types de soins comme s'ils étaient des corps vivants : ils étaient lavés, oints d'huile, parés et décorés de rubans et de couronnes fraîches. La décoration et l'entretien les rendaient agréables à la vue, lieux propices à recevoir des offrandes susceptibles de faire plaisir aux dieux et aux défunts. C'est cette idée de charme, d'éclat et de plaisir que les peintres attiques véhiculent à travers les motifs qui ornent leur surface. J'ai déjà parlé de l'autel figuré 
sur la coupe de Macron à Berlin (fig. 4); ici, je me limiterai à deux cas de monuments funéraires : une stèle funéraire et un sarcophage.

Figure 19

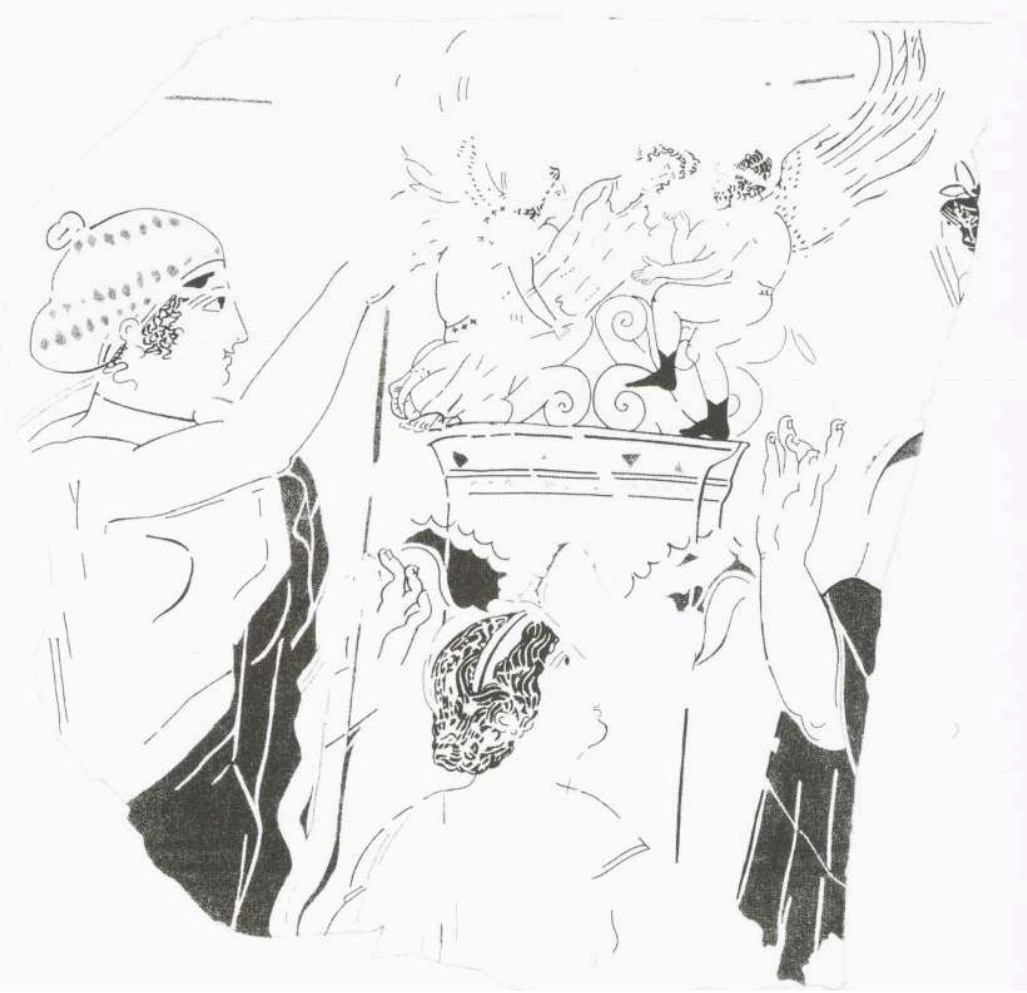

Lécythe à fond blanc, vers 460, Berlin, Antikensammlung 3325.

Sur un lécythe à fond blanc (vers 460$)^{94}$, malheureusement très fragmentaire, la stèle funéraire est le centre autour duquel gravitent plusieurs figures à des échelles différentes suggérant des réalités différentes (fig. 19). Au premier plan, une jeune femme est assise sur les degrés de la stèle : il s'agit de la défunte figurée sous les traits d'un vivant, image de ce qu'elle était autrefois mais qui reste invisible pour les visiteurs qui viennent lui rendre les honneurs. Il s'agit d'un homme et d'une femme, une bandelette à la main, et dont le regard se dirige vers le sommet de la stèle où l'on voit trois figures en miniature: les frères divins, Hypnos (Sommeil) et Thanatos (Trépas) déposant le corps inanimé de la défunte. S'agit-il d'une image sculptée faisant partie de l'acrotère ou d'une vision? Le peintre semble jouer sur l'ambiguïté. Quoi qu'il en soit, l'image miniaturisée embellit la stèle, elle-même substitut du corps du défunt : il suffit de noter le jeu graphique entre les ailes des deux frères et les feuilles d'acanthe figurées au milieu de la stèle. En même temps, l'image miniaturisée redouble la présence de la défunte, sur une autre échelle et sur un autre mode puisque son corps rigide ne manifeste aucune trace de vie. Enfin, elle est la seule à être vue, le regard de la femme à la bandelette en porte la preuve. 


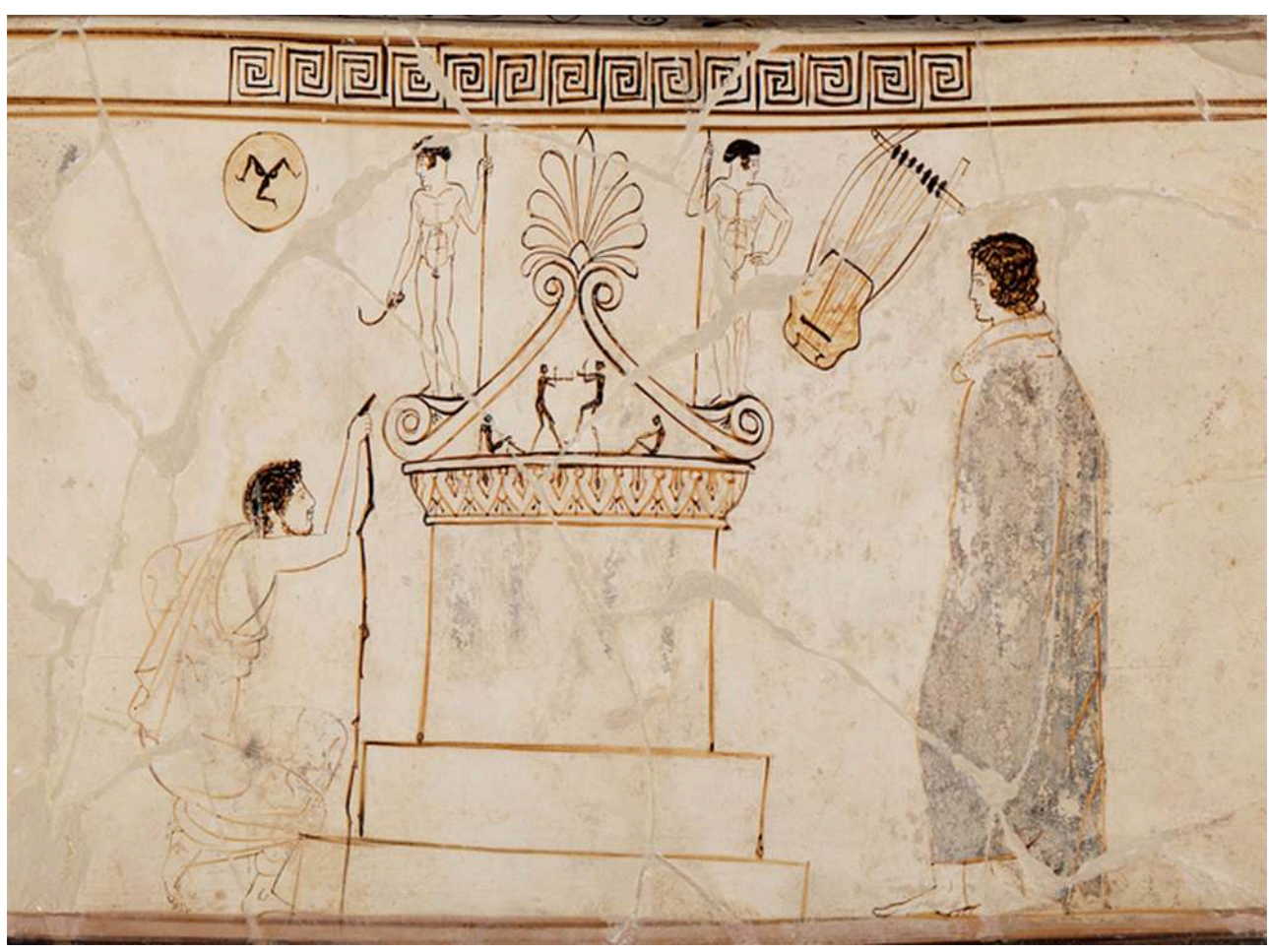

Lécythe à fond blanc attribué au Peintre de Thanatos, 450-440, Boston, Museum of Fine Arts 01.8080.

Enfin, pour couronner cette sélection, un lécythe à fond blanc du Peintre de Thanatos (450-440 $)^{95}$ célébrant le corps juvénile qui fut celui du défunt selon trois modalités différentes (fig. 20). D'abord, par la saynète de lutte qui orne le fronton du sarcophage dressé au milieu de l'image. Puis, par les deux statues d'athlètes, statues polyclétéennes qui servent d'acrotères au sarcophage. Et enfin, par l'image du disque figuré dans le champ de l'image : un triscèle (trois jambes) qui, tout comme sur les boucliers, véhicule l'idée de la rapidité. Ces trois représentations du corps viril ainsi que la lyre figurée dans le champ de l'image, évoquent la paideia, l'éducation, du défunt, sans doute le jeune homme qui se tient debout à droite du sarcophage.

Pour conclure: la mise en abyme plonge le regard dans une richesse à la fois esthétique, figurative et sémantique où chaque détail, chaque motif, aussi discret soitil, révèle une fonction précise et polyvalente au sein de l'image. Minutieusement exécutés, les motifs en abyme véhiculent l'idée de la bigarrure, de la brillance, du charme et du mouvement, valeurs qui font vibrer non seulement la surface de l'objet qu'ils ornent mais aussi la surface entière du vase. Cet effet de vie, porté par la poikilia et par la charis, vise avant tout à réjouir le spectateur. Or cette jouissance esthétique va de pair avec un plaisir intellectuel, puisque les motifs en abyme densifient le sens de l'image en créant plusieurs points de référence et niveaux de lecture qui peuvent fonctionner de manière complémentaire ou antithétique. Aucun doute que les catégories longuement dépréciées du «détail» et de l'«ornement» sont particulièrement signifiantes : grâce à elles, le peintre fait preuve d'une virtuosité technique, d'un savoir-faire de haut niveau qui rehausse la valeur du vase et fait de ce dernier un « objet de vision ». 


\section{NOTES}

1. Je me permets ici d'utiliser le terme "mise en abyme » au sens large du terme qui va bien audelà de la question du médium. Mon intérêt se porte principalement sur le langage visuel que les images et les motifs miniaturisés mobilisent et véhiculent au sein des images plus larges et plus complètes, langage identique à celui de l'ensemble de la céramique attique de l'époque archaïque et classique. Sur la mise en abyme voir en dernier lieu Jennifer TRIMBLE, « Figure and ornament, death and transformation in the Tomb of the Haterii » dans Nikolaus DIETRICH et Michael SQUIRE (éd.), Ornament and Figure in Graeco-Roman Art. Rethinking Visual Ontologies in Classical Antiquity, De Gruyter, Berlin-New York, 2018, p. 327-352 et dans le même ouvrage, Jaś ELSNER, "Ornament, figure and mise en abyme on Roman sarcophagi », p. 353-391.

2. Sur la poikilia voir Adeline GRAND-CLÉMENT, La fabrique des couleurs. Histoire du paysage sensible des Grecs anciens, Paris, De Boccard, 2011, p. 420-487.

3. Sur le kosmos voir à titre indicatif Michel CASEVITZ, "À la recherche du kosmos. Là tout n'est qu'ordre et beauté », Le temps de la réflexion X, 1989, p. 97-119, avec une riche bibliographie ; Clemente MARCONI, « Kosmos : The imagery of the Greek temple », RES : Anthropology and Aesthetics 45, 2004, p. 211-224.

4. Sur la notion de charis et ses nombreuses constellations voir entre autres Bonnie MACLACHLAN, The Age of Grace. Charis in Early Greek Poetry, Princeton, Princeton University Press, 1993 ; Carlo BRILLANTE, «Charis, bia e il tema della reciprocità amorosa », Quaderni Urbinati di Cultura Classica 59, 1998, p. 7-34 ; Vincent AZOUlAY, Xénophon et les grâces du pouvoir. De la charis au charisme, Paris, Publications de la Sorbonne, 2004; Joseph W. DAY, Archaic Greek Epigram and Dedication. Representation and Reperformance, Cambridge, Cambridge University Press, 2010, p. 232-280.

5. Françoise Frontisi-Ducroux, Dédale. Mythologie de l'artisan en Grèce ancienne, Paris, Ed. La Découverte, 2000 (1 $1^{\text {ère }}$ éd. 1975).

6. C'est la définition que propose J. W. DAY, Archaic Greek Epigram, op. cit., notamment p. 85-129. D'après l'auteur, le terme agalma, lorsqu'il se trouve dans les épigrammes dédicatoires, renvoie plutôt à l'effet esthétique et émotionnel que ce dernier est censé susciter chez le destinataire qu'à une catégorie particulière d'objets. Notons que le premier à avoir analysé la notion de valeur à la fois matérielle et immatérielle impliquée dans le terme "agalma » fut Louis GERNET, dans son article « La notion mythique de la valeur en Grèce », Journal de psychologie, 41, 1948, p. 415-462, repris dans Anthropologie de la Grèce antique, Paris, 1982, p.121-179. Sur l'agalma voir aussi Maria Letizia LAZZARINI, Le formule delle dediche votive nella Grecia arcaica, Rome, Accademia Nazionale dei Lincei, 1976, p. 95-98.

7. D’après Ioanna Patera n'est agalma que ce qui est nommé ainsi : «Agalma: le terme et le concept» dans Stéphan DUGAST, Dominique JAILlARD, Ivonne MANFRINI (dir.), Agalma ou les figurations de l'invisible. Approches comparées, Paris, éditions Jérôme Million, coll. "Horos", à paraître. Contrairement à Ioanna Patera, Nicole Lanérès estime que le terme « agalma » n'est pas toujours nécessaire pour désigner la chose : « La notion d'agalma dans les inscriptions grecques », Mètis, n. s. 10, 2012, p. 137-173 et notamment p. 154, n. 70.

8. Sur la catégorie de l'«ornement», je cite à titre indicatif le dossier intitulé «Ornement/ Ornemental » dans Perspective. La revue de l'INHA, n. 1, 2010-2011, traitant des déclinaisons, des définitions et des lectures de l'ornement et de l'ornemental de l'antiquité à nos jours. En ce qui concerne l'antiquité, voir entre autres les deux ouvrages collectifs du courant " néo-formaliste » : Verity PLATT, Michael squiRe (éd.), The Frame in Classical Art: A Cultural History, Cambridge, Cambridge University Press, 2017 et Nikolaus DIETRICH, Michael sQUiRE (éd.), Ornament and Figure, op.cit. Pour une riche bibliographie sur les études récentes autour de l'ornement en général, voir 
en particulier Michael SQUIRE, «'To haunt, to startle, and way-lay' : Approaching ornament and figure in Graeco-Roman art », dans Nikolaus DIETRICH, Michael SQUIRE (ed.), Ornament and Figure, op. cit., p. 16, n. 36.

9. Sur ce point voir Nikolaus DIETRICH, «Framing Archaic Greek Sculpture : Figure, Ornament and Script », dans Veritty PLATT, Michael SQUIRE (éd.), The Frame, op. cit., p. 270-316 et dans le même ouvrage, Clemente MARCONI, "The frames of Greek painted pottery », p. 117-153. Sur le rapport entre «ornement» et "figure» dans l'art gréco-romain en général voir Nikolaus DIETRICH, Michael SQUIRE (éd.), Ornament and Figure in Graeco-Roman Art, op.cit.

10. Sur le rapport interactif entre figures et motifs végétaux voir Nikolina KEI, "The Floral Aesthetics of Attic Red-figured Pottery : Visual Adornment and Interplay Between Ornament and Figure » dans Claudia LANG-AUINGER, Elisabeth TRINKL (éd.), $\Phi v \tau \alpha \kappa \alpha l ~ Z \omega l \alpha$. Pflanzen und Tiere auf Griechischen Vasen, (CVA Österreich, Beiheft 2), Vienne, 2016, p. 271-280.

11. Sur le détail voir Daniel ARASSE, Le détail. Pour une histoire rapprochée de la peinture, Paris, Flammarion, 1996, (1 ère éd. 1992).

12. Pour une approche sociologique des produits textiles voir Lloyd LLEWELLYN-JONES (éd.), Women's Dress in the Ancient Greek World, Londres/Swansea, Duckworth, 2002 ; John SCHEID et Jesper SVENBRo, Le métier de Zeus. Mythe du tissage et du tissu dans le monde gréco-romain, Paris, Éd. Errance, 2003 ; Liza CLELAND, Mary HARLOW, Lloyd LLEWELLYN-JONES (éd.), The Clothed Body in the Ancient World, Oxford, Oxbow Books, 2005 ; Hans VAN WEES, "Clothes, Class and Gender in Homer » dans Douglas CaIRNs (éd.), Body Language in the Greek and Roman Worlds, Swansea/Oakville, Classical Press of Wales, 2005, p.1-36; Florence GHERCHANOC, Valérie HUET (dir.), Vêtements antiques : s'habiller, se déshabiller dans les mondes anciens, (Actes des journées de recherches tenues entre le 31 mars 2007 et le 28 mars 2008, et d'un colloque international tenu le 26 et 27 novembre 2009), Paris, Éd. Errance, 2012 ; Florence GHERCHANOC, Valérie HUET (éds.), S’habiller, se déshabiller dans les mondes anciens, Mètis, n. s. 6, 2008, p. 5-158; Florence GHERCHANOC, Valérie HUET, « Le corps et ses parures dans l'Antiquité grecque et romaine : bilan historiographique ", Dialogues d'Histoire Ancienne, vol. S14 (Supplément 14), 2015, p. 127-150.

13. Dans l'Iliade (VI, 286-295), Hécube descend dans le thalamos odorant et choisit parmi les voiles (peploi) multicolores (pampoikila), confectionnés par des Sidoniennes, le plus beau par son riche décor coloré et le plus grand, celui qui brille comme un astre, afin de l'offrir à Athéna. Hélène choisit elle aussi le voile dans le thalamos royal, le plus grand et le mieux décoré, confectionné par elle-même et brillant comme un astre, pour l'offrir à Télémaque (Odyssée, XV, 105-130).

14. Iliade, III, 125-128.

15. John SCHEID, Jesper SVENBRO, Le métier de Zeus, op. cit., p. 96.

16. Iliade, VI, 289 ; Iliade, XXII, 440-441.

17. SAPPHO, fr. 1, 1 L-P. Les diverses interprétations de l'adjectif ont été reprises par Jacques JOUANNA, « Le trône, les fleurs, le char et la puissance d'Aphrodite », Revue des Études Grecques 112, 1999, p. 99-126 et notamment p. 101-3 ; John SCHEID, Jesper SVENBRo, Le métier de Zeus, op. cit., p. 51-72 ; Adeline GRAND-CLÉMENT, La fabrique des couleurs, op. cit., p. 459-463.

18. Londres, British Museum 1971.11-1.1 ; Para 19.16bis ; Add² 10 ; BA 350099.

19. Sur le décor floral des vêtements figurés sur les vases attiques voir Nikolina KEI, «Poikilia et kosmos floraux dans la céramique attique » dans Lydie BODIOU, Florence GHERCHANOC, Valérie HUET, Véronique MEHL (éds.), Parures et artifices: le corps exposé dans l'Antiquité gréco-romaine, Paris, L'Harmattan, 2011, p. 233-253.

20. Florence, Museo Archeologico Etrusco 4209 ; ABV 76.1, 682 ; Para 29 ; Add 21 ; BA 300000.

21. Paola COLAFRANCESCHI CECCHETTI, Decorazione dei costumi nei vasi attici a figure nere, (Studi Miscellanei XIX), Rome, De Luca, 1972, pl. XVIII. 44. 
22. La coupe est attribuée à Onésimos ; Rome, Museo Nazionale Etrusco di Villa Giulia 121110 ; BA 13363.

23. Voir Beth COHEN, «The Anatomy of Kassandra's Rape: Female Nudity Comes of Age in Greek Art » dans Essays on Nudity in Antiquity in Memory of Otto Brendel, New York, (Source. Notes in the History of Art 12, n. 2), 1993, p. 37-46 ; Joan Breton ConNeLLY, « Narrative and Image in Attic Vase Painting. Ajax and Kassandra at the Trojan Palladion » dans Peter James HOLLIDAY (éd.), Narrative and Event in Ancient Art, Cambridge, Cambridge University Press, p. 88-129.

24. Londres, British Museum E140; ARV² 459.3, 481, 1654 ; Para 377 ; Add ${ }^{2} 243$; BA 204683.

25. Sur la poikilia des écailles et de la peau du serpent voir Adeline GRAND-CLÉMENT, La fabrique des couleurs, op. cit., p. 444-447.

26. Sur l'imagerie maritime du symposion, voir François LISSARRAGUE, Un flot d'images. Une esthétique du banquet grec, Paris, A. Biro, 1987, p. 104-118.

27. Berlin, Antikensammlung F2290; ARV² 462.48, 481, 1654 ; Para 377 ; Add 244 ; BA 204730.

28. Sur ce point voir Eric CSAPO, "The Dolphins of Dionysos" dans Eric CSAPO et Margaret Christina MILLER (éds.), Poetry, Theory, Praxis: The Social Life of Myth, Word and Image in Ancient Greece. Essays in Honour of William J. Slater, Oxford, Oxbow Books, 2003, p. 69-98.

29. Bâle, Antikenmuseum und Sammlung Ludwig Bs415;BA 260.

30. Des lettres inscrites près de leur bouche indiquent qu'ils sont en train de chanter.

31. Des chitoniskoi au décor floral sont également portés par des danseurs sur une hydrie du Peintre de Pan (Saint Petersburg, Musée de l'Ermitage 627 ; ARV²555.95, 481, 1654 ; Para 387, 388 ; Add $^{2} 258$; BA 206338).

32. Sur le caractère dionysiaque de la performance voir Matthew C. WELLENBACH, "The Iconography of Dionysiac Choroi : Dithyramb, Tragedy, and the Basel Krater ", Greek, Roman, and Byzantine Studies 55, 2015, p. 72-103 et particulièrement sur le rôle du costume comme élément d'individualisation, p. 93.

33. Chiusi, Museo Archeologico Nazionale 1831; ARV²1300.2, 1689 ; Para 475 ; Add 360 ; BA 216789.

34. Outre ses ailes, l'homme porte des bottes ailées, ce qui redouble sa vélocité.

35. L'ouvrage est censé être un vêtement funéraire destiné au père d'Ulysse, Laërte.

36. Sur ce point voir John SCHEID, Jesper SVENBRo, Le métier de Zeus, op. cit., p. 59 ; Sheramy D. BUNDRICK, « The Fabric of the City », Hesperia, 77, n. 2, 2008, p. 283-334, et notamment p. 324.

37. Sur ce point voir François LISSARRAGUE, «Corps et armes : figures grecques du guerrier» dans Véronique DASEN, Jérôme WILGAUX (éd.), Langages et métaphores du corps dans le monde antique, Rennes, Presses Universitaires de Rennes, 2008, p.15-27. Voir aussi dans le même ouvrage, l'article de Véronique MEHL, «Corps iliadiques, corps héroïques », p. 29-42.

38. Sur cette double facette des armes et sur leurs vertus surnaturelles voir Françoise FRONTISIDucroux, Dédale, op. cit., p. 65-68.

39. Sur les ornements des armes voir François LISSARRAGUE, « Armure et ornement dans l'imagerie attique » dans Nikolaus DIETRICH et Michael SQUIRE (éd.), Ornament and Figure, op. cit., p. 129-141. Sur le décor floral des armes voir Nikolina KEI, L'esthétique des fleurs: kosmos, poikilia et charis dans la céramique attique du $V^{e}$ et $V^{e}$ siècle av. J.-C., thèse soutenue à l' EHESS, Paris, 2010, p. 84-97.

40. Sur le spectre sémantique de l'adjectif « aiolos » voir Marcel DETIENNE, Jean-Pierre VERNANT, Les

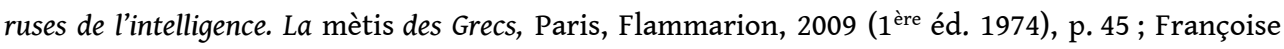
FRONTISI-DUCROUX, Dédale, op. cit., p. 69 ; Adeline GRAND-CLÉMENT, La fabrique des couleurs, op. cit., p. 83-91.

41. Sur les divers types d'épisèmes que l'on trouve dans les images et les textes grecs voir George H. CHASE, The Shield Devices of the Greeks in Art and Literature, Chicago, Ares, 1979 (Cambridge 1902). Il existe aussi le mémoire de maîtrise non publié d'Anne JACQUEMIN intitulée «Les épisèmes de boucliers ", soutenu à Paris IV, en Juin 1973. 
42. Rappelons que c'est l'épisème du bouclier qui permet d'identifier son détenteur et, à la fin du $\mathrm{V}^{\mathrm{e}}$ siècle, le camp auquel il appartient.

43. Iliade, XVIII, $468-617$.

44. Pour une analyse détaillée de la fonction des épisèmes dans le déroulement de l'intrigue voir Froma ZEITLIN, Under the Sign of the Shield: Semiotics Aeschylus' Seven Against Thebes, Rome, Éd. dell'Atenco, 1982 et Pierre VIDAL-NAQUET, « Les boucliers des héros. Essai sur la scène centrale des Sept contre Thèbes", dans Jean-Pierre VERNANT, Pierre VIDAL-NAQUET, Mythe et tragédie en Grèce ancienne, vol. 2, Paris, 2001 (1 ère éd. 1988), p. 115-47. Sur le rapport entre représentation visuelle et poétique des épisèmes voir Cécile JUBIER-GALINIER, Annie-France LAURENS, «Boucliers en images et images de bouclier, effets de réel » dans Pierre SAUZEAU, Thierry VAN COMPERNOLLE (éd.), Les armes dans l'antiquité. De la technique à l'imaginaire, Montpellier, Presses universitaires de la Méditerranée, 2007, p. 105-120 ; François LISSARRAGUE, «Looking at Shield Devices : Tragedy and Vase Painting » dans Christina Sh. KRAUS, Simon GOLDHILL, Helene P. FOLEY, Jas ELSNER Visualizing the Tragic. Drama, Myth and Ritual in Greek Art and Litarature: Essays in Honor of Froma Zeitlin, Oxford, Oxford University Press, 2007, p. 151-64 et id., «Le temps des boucliers » dans Giovanni CARERI, François LISSARRAGUE, Jean-Claude SCHMITT et Carlo SEVERI (éds.), Traditions et temporalités des images, Paris, EHESS, 2009, p. 21-31, version en ligne dans Images Re-vues, H.S. 1, 2008 (http:// journals.openedition.org/imagesrevues/850), consulté le 09 juillet 2016.

45. Londres, British Museum B329 ; ABV 334.1, 678 ; Para 147, 388 ; Add 91 ; BA 301814.

46. Sur le lion et les fauves dans les épopées homériques voir Annie SCHNAPP-GOURBEILLON, Lions, Héros, masques : les représentations de l'animal chez Homère, Paris, F. Maspero, 1981, p. 39 sq ; Adeline GRAND-CLÉMENT, La fabrique des couleurs, op. cit., p. 303-305.

47. PINDARE, Olympiques, IX, 42.

48. Iliade, XIX, 121; XX, 16; XXII, 178; PINDARE, Olympiques, VIII, 3. Sur ces deux épithètes voir Adeline GRAND-CLÉMENT, La fabrique des couleurs, op. cit., p. 90.

49. ESCHYLE, Prométhée Enchaîné, 7-8. Pour autres références littéraires voir Paul JACOBSTHAL, Der Blitz in der orientalischen und griechischen Kunst, Berlin, Weidmannsche Buchhandlung, 1906, p. 10 sq. et Arthur Bernard соок, Zeus. A Study in Ancient Religion: God of the Dark Sky (thunder and lightning), Cambridge, 1925, p. 771, n. 3.

50. Sur les formes florales du foudre voir Paul JACOBSTHAL, Der Blitz in der orientalischen und griechischen Kunst, op. cit.

51. Malibu, J. P. Getty Museum 86.AE.306.1-4 ; BA 275034 ; CVA Malibu, J. Paul Getty Museum 8, p. 13-14, pl. 397.2.4-6.

52. Sur la mêtis du poulpe voir «Le renard et le poulpe » dans Marcel DETIENNE, Jean-Pierre VERNANT, Les ruses de l'intelligence, op. cit., p. 32-57.

53. Sur le poulpe dans la céramique grecque voir Nikolina KEI, « La mêtis du décor : de la palmette au poulpe ", dans Vasiliki ZACHARI, Élise LEHOUX, Noémie HOSOI (dir.), La cité des regards. Autour de François Lissarrague, Presses universitaires de Rennes, 2019, p. 217-228.

54. New York, Metropolitan Museum of Art 08.258.58; ARV 185.36 ; Para 340 ; Add $^{2} 187$; BA 201688.

55. Sur le lézard dans la céramique voir Ingrid EDLUND, « Meaningful or Meaningless? Animal Symbolism in Greek Vase Painting ", Mededelingen van het Nederlands Instituut te Rome, 42, n. s. 7, p. 31-35 ; Jeffrey M. HURWIT, « Lizards, lions, and the uncanny in Early Greek Art », Hesperia, 75, 2006, p. 121-136.

56. Munich, Antikensammlungen $2688 ; A R V^{2}$ 879.1, 1673; Para 428; Add 200 ; BA 211565.

57. Sur la fonction déictique des motifs floraux sur certains vases voir Nikolina Kei, «The Floral Aesthetics ... », op. cit., p. 272-73.

58. Sur le mobilier antique en général voir Gisela M. A. RICHTER, The Furniture of the Greeks, Etruscans and Romans, Londres, Phaidon P., 1966. Voir aussi Helmut JUNG, Thronende und sitzende 
Götter. Zum griechischen Götterbild und Menschenideal in geometrischer und früharchaischer Zeit, Bonn, R. Habelt, 1982 ; Helmut KYRIELEIS, Throne und Klinen : Studien zur Formgeschichte altorientalischer und griechischer Sitz- und Liegemöbel vorhellenistischer Zeit, Berlin, De Gruyter, 1969.

59. Iliade, XVIII, 389-390, d'après la traduction de Paul MAZON (CUF).

60. PAUSANIAS, Description de la Grèce, $\mathrm{V}, 11,2$. Nous suivons la traduction de Jean Pouilloux (CUF) à l'exception du terme « agalmata » traduit par « statues".

61. Sur le sujet voir Nassi MALAGARDIS, «Héra, la sans pareille ou l'épouse exclue ? À travers l'image ", dans Juliette de LA GENIÈrE (éd.), Héra. Images, espaces, cultes. Actes du Colloque International de Lille III et de l'Association PRAC, Lille, 29-30 novembre 1993, Collection du Centre Jean Bérard 15, Naples, Centre Jean Bérard, 1997, p. 93-111.

62. Londres, British Museum B147 ; ABV 135.44 ; Para 55 ; Add ${ }^{2} 36$; BA 310304.

63. Lion : amphore, Peintre de Princeton, Génève, Musée d'Art et d'Histoire MF154 ; ABV 299.18 ; Para 130; $A d d^{2} 78$; BA 320417. Deux lions sur une plante fleurie: amphore, Groupe E, Bâle, Antikenmuseum und Sammlung Ludwig 103.4 ; LIMC II, «Athena », n. 353, pl. 745 ; BA 213.

64. Hydrie, Rome, Musei Capitolini 65 ; CVA, Roma, Musei Capitoloni 1, III.H.12, pl. 26.2 ; BA 100.

65. Amphore, Groupe E, Munich, Antikensammlungen 1382 ; ABV 135.47 ; Add $^{2} 36$; BA 310307.

66. Pyxide, Peintre C, Paris, Musée du Louvre CA616; ABV 58.122 ; Para 23 ; Add ${ }^{2} 16$; BA 300499.

67. Amphore, Groupe E, Paris, Musée du Louvre F32 ; ABV 135.43 ; Para 55 ; Add A $^{2} 36$; BA 310303.

68. Amphore, proche du Groupe E, Vienne, Kunsthistorisches Museum 3596 ; ABV 138.1, 136 ; Para 56.48bis, 57.1 ; Add $^{2} 37$; BA 310333.

69. Amphore, Groupe E, Boston, Museum of Fine Arts 00.330 ; ABV 135.45 ; Para 55 ; Add $^{2} 36$; BA 310305 et Amphore, Peintre de Princeton, Madrid, Museo Arqueologico Nacional 1999.99.52 ; BA 29025.

70. Amphore, Groupe E, Philadelphie, University of Pensylvania 3440 ; LIMC III, «Eileithyia », n. 18, pl. 535 ; BA 14652. Dans la céramique à figures rouges, une amphore du Peintre de Nikoxenos (Munich, Antikensammlungen 2304, $A R V^{2}$ 220.1; Para 346; Add ${ }^{2} 198$; BA 202095) représente un trône " animé » où deux sphinx sont sculptés à la place des accoudoirs, alors que deux éphèbes nus, en position de lutte, soutiennent le siège.

71. Paris, Cabinet des Médailles 418 ; ARV² 260.2, 1640 ; Para 351 ; Add 204 ; BA 202956.

72. Le plongeon, preuve de la filiation divine de Thésée, est raconté par Bacchylide dans son $17^{\mathrm{e}}$ Dithyrambe (vers 470 av. J.-C.).

73. J'adopte le terme que Jeffrey M. Hurwit a utilisé lors de sa communication «Elements of Nature in Archaic Greek Art: The Sea », présentée dans le cadre d'une table ronde intitulée «La représentation de la nature dans l'art grec de l'époque archaïque à l'époque hellénistique », à l'INHA, à Paris, 18 mai 2012. Sur la représentation de l'espace maritime voir Jeffrey M. HURWIT, "The Representation of Landscape in Early Greek Art» dans Diana BUITRON-OLIVER (éd.), New Perspectives in Early Greek Art, (Studies in the History of Art, vol. 32), Washington D. C., National Gallery of Art, University Press of New England, 1991, p. 33-62 et plus récemment, Nikolaus DIETRICH, Figur ohne Raum ?, Berlin/New York, Walter De Gruyter, 2010, p. 22-34.

74. BACCHYLIDE, Dithyrambe, 17.97-100 Loeb/Snell-Maehler.

75. On retrouve des dauphins « réels" sur l'intérieur d'une coupe d'Euphronios (potier) et attribuée à Onésimos représentant Thésée accueilli par Amphitrite dans son palais maritime (Paris, Musée du Louvre G104 ; ARV² 318.1, 1645, 313 ; Para 358 ; Add² 214 ; BA 203217).

76. Voir Gisela M. A. RICHTER, The Furniture of the Greeks, Etruscans and Romans, op. cit., p. 52-63.

77. Le lit d'Ulysse et de Pénélope a été fait d'or, d'argent et d'ivoire plaqués sur du bois d'olivier : Odyssée, XXIII, 200.

78. Bruxelles, Musées Royaux A717; ARV² 20.1, 1619 ; Para 322 ; Add ${ }^{2} 154$; BA 200102.

79. Sur l'iconographie de la rançon d'Hector dans la céramique attique voir surtout Anneliese KosSATZ-DEISSMANN, s. v. « Achilles », dans Lexicon Iconographicum Mythologiae 
Classicae, I, Zurich, Munich, Artemis, 1981, vol. 1, p. 37-200 et vol. 2, p. 56-145 ; Odette TOUCHEFEU, s. v. " Hektor ", dans Lexicon Iconographicum Mythologiae Classicae, IV, Zurich, Munich, Artemis, 1988, vol. 1, p. 482-498 et vol. 2, p. 283-291; Wolfgang BASISTA, "Hektors Lösung », Boreas 2, p. 5-36. Luca GIULIANI, Image and Myth: a history of pictorial narration in Greek art, Chicago/London, University of Chicago Press, 2013, (éd. orig. München, 2003), p. 139-156 ; Nikolina KÉI, François LISSARRAGUE, « Aglaa dôra: la rançon d'Hector et le système des objets » dans Stéphan Dugast, Dominique Jaillard, Ivonne Manfrini (dir.), Agalma ou les figurations de l'invisible. Approches comparées, op. cit. ; Odette TOUCHEFEU, «L'humiliation d'Hector », Métis 5, 1990, p. 157-168 ; Marta PEDRINA, La supplication sur les vases grecs : mythes et images, Pisa/Roma, Fabrizio Serra editore, 2017, p. 341-352.

80. Vienne, Kunsthistorisches Museum 328 ; $A R V^{2}$ 380.171, 1649 ; Para 366, 368; Add $^{2} 227$; BA 204068.

81. Iliade, XXII, 93-5 : « Tel un serpent des montagnes, sur son trou, attend l'homme, il s'est repu de poisons malfaisants, une colère atroce le pénètre; il regarde d'un œil effrayant, lové autour de son trou ». Sur le serpent voir Annie SCHNAPP-GOURBEILLON, Lions, Héros, masques, op. cit., p. 91.

82. Iliade, XXI, 573-4 ; Annie SCHNAPP-GOURBEILLON, Lions, Héros, masques, op. cit., p. 89.

83. Iliade, XVII, 542 .

84. François LISSARRAGUE, Vases grecs. Les Athéniens et leurs images, Paris, Hazan, 1999, p. 98.

85. Munich, Antikensammlungen 2618 ; ARV 61.74, 1622 ; Para 327 ; Add ${ }^{2} 165$; BA 200510.

86. Le jeu de la "réflexivité " a été abordé par François Lissarrague dans son livre, Un flot d'images. Une esthétique du banquet grec, op. cit., et surtout dans son article « Réflexions sur l'image dans la céramique de Grande Grèce » dans Gemma SENA CHIESA (éd.), Vasi, immagini, collezionismo : giornate di studio : la collezione di vasi Intesa SanPaolo e i nuovi indirizzi di ricerca greca e magnogreca, Milano 7-8 novembre 2007, Milan, CISALPINO, 2008, p. 209-227. Il existe également l'étude de Helga GERICKE, Gefässdarstellungen auf griechischen Vasen, Berlin, B. Hessling, 1970 et deux articles, celui de Werner OENBRINK, « Ein “Bild im Bild”-Phänomen - Zur Darstellung figürlich dekorierter Vasen auf bemalten attischen Tongefäßen », Hephaistos 14, 1996, p. 81-134, et celui de Marjorie Susan VENIT, « Point and Counterpoint. Painted Vases on Attic Painted Vases », AK 49, 2006, p. 29-41. M. $\mathrm{S}$. Venit préfère le terme « réitération » à celui de « réflexion ».

87. New York, Metropolitan Museum of Art 1997.388a-eee; 1997.493 ; 1996.56a ; BA 46026. Pour une analyse plus approfondie voir Mary MOORE, « Hephaistos Goes Home: An Attic Black-figured Column-krater in the Metropolitan Museum ", Metropolitan Museum Journal, 45, 2010, p. 21-54 ; Guy Michael HEDREEN, The Image of the Artist in Archaic and Classical Greece: Art, Poetry, and Subjectivity, New York, Cambridge University Press, 2016, p. 212-217.

88. Des rosaces ornent parfois la lèvre des vases (cratères et amphores) datant de la première moitié du VI ${ }^{\mathrm{e}}$ siècle.

89. Pour une analyse plus approfondie de ces deux images voir Werner OENBRINK, «Ein "Bild im Bild"-Phänomen- Zur Darstellung figürlich dekorierter Vasen auf bemalten attischen Tongefäßen », art.cit., p. 100 sq. ; Marjorie Susan VENIT, «Point and Counterpoint. Painted Vases on Attic Painted Vases ", art.cit., p. 32-33, pl. 7.1-4.

90. François LISSARRAGUE, Un flot d'images. Une esthétique du banquet grec, op. cit., p. 98.

91. Vicence, Palazzo Leoni Montanari ; ARV²571.73, 1659; Para 390 ; Add 261 ; BA 206564.

92. On trouve le terme sur une coupe des Petits Maîtres datant de la deuxième moitié du VI ${ }^{\mathrm{e}}$ siècle, consacrée par un certain Nouménios à Athéna sur l'Acropole d'Athènes (Maria Letizia LAZZARINI, Le formule delle dediche votive nella Grecia arcaica, op. cit., n.710) ainsi que sur le pied d'un vase daté $\mathrm{du} \mathrm{V}^{\mathrm{e}}$ siècle, également consacré sur l'Acropole d'Athènes, où il est explicitement indiqué qu'il a été fait savamment (Maria Letizia LAZZARINI, Le formule delle dediche votive nella 
Grecia arcaica, op. cit., n. 822 ; ABV 351). La question de la valeur des vases en terre cuite a soulevé des débats vifs parmi les spécialistes. La thèse de Michael VICKERS, David GILL (Artful Crafts : Ancient Silverware and Pottery, Oxford/New York, Oxford University Press, 1995) selon laquelle les vases en terre cuite étaient des produits de qualité douteuse, pauvres imitations des vases métalliques utilisés par l'élite au cours des banquets et autres occasions, a connu plusieurs réfutations, parmi lesquelles figurent celles de John BOARDMAN ("Silver is White», Revue Archéologique, 1987, p. 279-95), Dyfri williams («Refiguring Attic Red-figure : A Review Article», Revue Archéologique, 1996, p. 227-52) et Richard T. NEER (Style and politics in Athenian vase-painting. The craft of democracy, ca. 530-460 B. C. E., Cambridge, Cambridge University Press, 2001, p. 206-15). Voir aussi Annie VERBANCK-PIERARD, "Sous les yeux d'Athéna et des Athéniens: vases, techniques et statut de l'artisan à l'Acropole ", Mètis, n. 12, 2014, p. 25-49.

93. Sur cette notion de «constructeur d'espace» voir Jean-Louis DURAND, François LISSARRAGUE, « Un lieu d'image ? L'espace du louterion », Hephaistos, 2, 1980, p. 89-106.

94. Berlin, Antikensammlung 3325 ; BA 46170. Sur ce lécythe voir Efthymia MINTSI, « Hypnos et Thanatos sur les lécythes attiques à fond blanc (deuxième moitié du Ve siècle av. J.-C.) ", Revue Études Anciennes 99, 1997, n. 1-2, p. 47-61, notamment p. 55 sq.

95. Boston, Museum of Fine Arts 01.8080 ; ARV 1231 ; BA 216394 ; John H. OAKLEY, Picturing Death in Classical Athens, New York, Cambridge University Press, 2004, p. 192.

\section{RÉSUMÉS}

Cet article s'intéresse à la mise en abyme de ce qu'on appelle traditionnellement "ornements » sur un certain nombre de vases attiques : il s'agit des motifs végétaux, animaliers, stellaires et même des figures humaines, représentés de manière micrographique sur des objets de luxe meublant des scènes à caractère varié. La présence discrète de ces motifs, loin d'être superflue, est d'ordre dynamique : ils opèrent à la fois comme ornements et signes polyvalents. En tant qu'ornements, ils véhiculent l'idée de la bigarrure, de la brillance, du charme et du mouvement, valeurs qui font vibrer non seulement la surface de l'objet qu'ils ornent mais aussi la surface entière du vase. En tant que signes, ils renvoient à l'ensemble de l'image, que ce soit sous forme d'écho, de répétition, d'intensification, de prolongement, d'explicitation ou de contraste. La jouissance esthétique va ainsi de pair avec le plaisir intellectuel tandis que les catégories longuement dépréciées $d u$ «détail» et de l'«ornement» se révèlent particulièrement signifiantes.

This paper considers the "mise en abyme " of what is traditionally called "ornaments" on a certain number of Attic vases: these are vegetal, animal, stellar motifs and even human figures, represented micrographically on luxury objects furnishing scenes of a varied nature. The discreet presence of these motifs, far from being superfluous, is dynamic : they operate as both ornaments and polyvalent signs. As ornaments, they convey the idea of variegation, brilliance, charm and movement, values which make vibrate not only the surface of the object they adorn but also the entire surface of the vase. As signs, they refer to the whole image, whether in the form of echo, repetition, intensification, extension, clarification or contrast. Aesthetic enjoyment thus goes hand in hand with intellectual pleasure, while the long-depreciated categories of "detail" and "ornament" are being proved to be particularly significant. 
INDEX

Keywords : Attic vases, ornaments, kosmos, charis, poikilia, daidalon, agalma

Mots-clés : Céramique attique, ornements, kosmos, charis, poikilia, daidalon, agalma

\section{AUTEUR}

\section{NIKOLINA KEI}

Ingénieure de recherche en analyse des images du monde gréco-romain à l'EHESS, attachée à l'UMR 8210 -ANHIMA, enseignante-vacataire à Paris 1-Sorbonne (UFR03, Histoire de l'art et archéologie) et co-responsable du séminaire « Vases grecs : images, corpus, collections » à l'INHA. Mes recherches portent principalement sur l'imagerie de la céramique attique, avec un intérêt particulier pour le rapport entre figures et « ornements ». 